\title{
Renormalization of Critical Gaussian Multiplicative Chaos and KPZ formula
}

\author{
Bertrand Duplantier, Rémi Rhodes $\dagger$ Scott Sheffield; \\ and VINCENT VARGAS ${ }^{\S}$ \\ * Institut de Physique Théorique, CEA/Saclay \\ F-91191 Gif-sur-Yvette Cedex, France \\ † Université Paris-Dauphine, Ceremade, UMR 7564 \\ Place du Maréchal de Lattre de Tassigny \\ 75775 Paris Cedex 16, France \\ $\ddagger$ Department of Mathematics \\ Massachusetts Institute for Technology \\ Cambridge, Massachusetts 02139, USA
}

February 20, 2013

\begin{abstract}
Gaussian Multiplicative Chaos is a way to produce a measure on $\mathbb{R}^{d}$ (or subdomain of $\mathbb{R}^{d}$ ) of the form $e^{\gamma X(x)} d x$, where $X$ is a log-correlated Gaussian field and $\gamma \in[0, \sqrt{2 d})$ is a fixed constant. A renormalization procedure is needed to make this precise, since $X$ oscillates between $-\infty$ and $\infty$ and is not a function in the usual sense. This procedure yields the zero measure when $\gamma=\sqrt{2 d}$.

Two methods have been proposed to produce a non-trivial measure when $\gamma=$ $\sqrt{2 d}$. The first involves taking a derivative at $\gamma=\sqrt{2 d}$ (and was studied in an earlier paper by the current authors), while the second involves a modified renormalization scheme. We show here that the two constructions are equivalent and use this fact to deduce several quantitative properties of the random measure. In particular, we complete the study of the moments of the derivative martingale, which allows us to establish the KPZ formula at criticality.
\end{abstract}

*e-mail: bertrand.duplantier@cea.fr. Partially supported by grant ANR-08-BLAN-0311-CSD5 and by the MISTI MIT-France Seed Fund.

$\dagger$ e-mail: rhodes@ceremade.dauphine.fr. Partially supported by grant ANR-11-JCJC.

${ }_{\ddagger}^{\ddagger}$-mail: sheffield@math.mit.edu. Partially supported by NSF grants DMS 064558, OISE 0730136 and DMS 1209044, and by the MISTI MIT-France Seed Fund.

$\S$ e-mail: vargas@ceremade.dauphine.fr. Partially supported by grant ANR-11-JCJC. 


\section{Introduction}

\subsection{Overview}

In the eighties, Kahane [34] developed a continuous parameter theory of multifractal random measures, called Gaussian multiplicative chaos. His efforts were followed by several authors $[2,5,7,17,26,51,52,55]$ coming up with various generalizations at different scales. This family of random fields has found many applications in various fields of science, especially in turbulence and in mathematical finance.

Roughly speaking, a Gaussian multiplicative chaos on $\mathbb{R}^{d}$ or on a bounded domain of $\mathbb{R}^{d}$ (with respect to the Lebesgue measure) is a random measure that can formally be written as:

$$
M(d x)=e^{\gamma X(x)-\frac{\gamma^{2}}{2} \mathbb{E}\left[X(x)^{2}\right]} d x
$$

where $X$ is a centered Gaussian distribution and $\gamma$ a nonnegative parameter. The situation of interest is when the field $X$ is log-correlated, that is when

$$
K(x, y) \stackrel{\text { def }}{=} \mathbb{E}[X(x) X(y)]=\ln \frac{1}{|x-y|}+g(x, y)
$$

for some bounded continuous function $g$. In this case, $X$ is a Gaussian random generalized function (a.k.a. distribution) on $\mathbb{R}^{d}$ that cannot defined as an actual function pointwise. Kahane showed that one can nonetheless give a rigorous definition to (1). Briefly, the idea is to cut off the singularity of the kernel (2) occurring at $x=y$ (sometimes referred to as ultraviolet cutoff). The cut-off strategy we will use here is based on a white noise decomposition of the Gaussian distribution $X$. In short, we will assume that the covariance kernel of $X$ can be written as:

$$
K(x, y)=\int_{1}^{+\infty} \frac{k(u(x-y))}{u} d u
$$

for some continuous covariance kernel $k$. Though not covering the whole family of kernels of type (2), this family of fields is quite natural since it possesses nice scaling relations (see [2]). A white noise decomposition of $X$ involves formally writing $X$ as

$$
X(x)=\int_{1}^{\infty} \int_{\mathbb{R}^{d}} \frac{g(y+x)}{\sqrt{s}} W(d s, d y)
$$

where $W$ is a space-time white noise and $g$ is a convolution square root of $k$. Then a cut-off of $X$ at level $t$ can be written as

$$
X_{t}(x)=\int_{1}^{e^{t}} \int_{\mathbb{R}^{d}} \frac{g(y+x)}{\sqrt{s}} W(d s, d y) .
$$

This is a Gaussian field with continuous covariance kernel that approximates $X$ in the sense that we recover the distribution $X$ when letting $t$ go to $\infty$. Having applied this cutoff, it is now possible to define the approximate measure

$$
M_{t}(d x)=e^{\gamma X_{t}(x)-\frac{\gamma^{2}}{2} \mathbb{E}\left[X_{t}(x)^{2}\right]} d x
$$


The family $\left(M_{t}\right)_{t}$ is a positive martingale, so it converges almost surely. The measure $M$ is then understood as the almost sure limit of this martingale. The limiting measure $M$ is non trivial if and only if $\gamma^{2}<2 d$ (see [34]). For $\gamma^{2} \geqslant 2 d$, the measure $M$ as defined by (1) thus vanishes, giving rise to the issue of constructing non trivial objects for $\gamma^{2} \geqslant 2 d$ in any other possible way.

In this paper, we pursue the effort initiated in [17] to understand the critical case, that is when $\gamma^{2}=2 d$. It is shown in [17] that the natural object at criticality is the derivative multiplicative chaos, which can be formally written as

$$
M^{\prime}(d x)=\left(\sqrt{2 d} \mathbb{E}\left[X(x)^{2}\right]-X(x)\right) e^{\sqrt{2 d} X(x)-d \mathbb{E}\left[X(x)^{2}\right]} d x .
$$

It is a positive atomless random measure. It can be rigorously defined via cut-off approximations in the same spirit as in (3). More precisely, the approximations are obtained by differentiating (3) with respect to $\gamma$ at the value $\gamma^{2}=2 d$, hence the term "derivative". Nevertheless, it is expected that derivative Gaussian multiplicative chaos at criticality, that is $M^{\prime}$, can be recovered via a properly renormalized version of (3). In this paper, inspired by analog results in the case of branching Brownian motion [44] or branching random walk [1], we prove that

$$
\sqrt{t} M_{t}(d x) \rightarrow \sqrt{\frac{2}{\pi}} M^{\prime}(d x), \quad \text { in probability as } t \rightarrow \infty .
$$

This renormalization procedure is sometimes called Seneta-Heyde scaling [1]. Property (5) establishes the important fact that the derivative martingale also appears as the limit of the natural renormalization of the vanishing martingale (3) for $\gamma^{2}=2 d$. Beyond this unifying perspective, this renormalization approach to criticality is convenient to complete the description of the main properties of the derivative martingale initiated in [17]. In particular, the Seneta-Heyde renormalization turns out to be crucial to applying Kahane's convexity inequalities at criticality. From this, we complete the study of the moments of the derivative martingale and compute its power-law spectrum. These properties are a prerequisite for establishing the celebrated Knizhnik-Polyakov-Zamolodchikov (KPZ) formula at criticality arising in Liouville quantum gravity. Let us stress that our proof of the KPZ formula shows that it is valid for any log-correlated Gaussian field for which one can prove a critical Senete-Heyde renormalization theorem of the type (5).

Recently, the authors in [20] constructed a probabilistic and geometrical framework for two dimensional Liouville quantum gravity and the KPZ equation [39], based on the two-dimensional Gaussian free field (GFF) (see [14, 15, 16, 20, 27, 39, 48] and references therein for physics considerations). It consists in taking $X$ equal to the GFF in (1). In this context, the KPZ formula has been proved rigorously [20, 53] (see also [10] in the context of Mandelbrot's multiplicative cascades). This was done in the standard case of Liouville quantum gravity, namely strictly below the critical value of the GFF coupling constant $\gamma$ in the Liouville conformal factor, i.e, for $\gamma^{2}<4$ (recall that the phase transition occurs at $\gamma^{2}=2 d$ in dimension $d$, producing a phase transition for $\gamma^{2}=4$ in dimension 2). 


\subsection{Extensions of our results: the Liouville measure at critical- ity in dimension 2}

Up to minor modifications, the techniques we developped in [17] and the present paper enable to prove analogous results in the case where $X$ is the GFF on a bounded domain $D \subset \mathbb{R}^{2}$. Thus we can give a rigourous definition to the standard Liouville measure at criticality (see discussion below). More precisely, our techniques apply straightforwardly within the following approach. The Green function is given by the formula:

$$
G_{D}(x, y)=\pi \int_{0}^{\infty} p_{D}(t, x, y) d t
$$

where $p_{D}$ is the (sub-Markovian) semi-group of a Brownian motion $B$ killed upon touching the boundary of $D$, namely

$$
p_{D}(t, x, y)=P^{x}\left(B_{t} \in d y, T_{D}>t\right)
$$

with $T_{D}=\inf \left\{t \geqslant 0, B_{t} \notin D\right\}$. Note that the $\pi$ term ensures that $G_{D}(x, y) \underset{|x-y| \rightarrow 0}{\sim}$ $-\ln |x-y|$. The most direct way to construct the GFF is then to consider a white noise $W$ distributed on $D \times \mathbb{R}_{+}$and define:

$$
X(x)=\sqrt{\pi} \int_{D \times \mathbb{R}_{+}} p_{D}\left(\frac{s}{2}, x, y\right) W(d y, d s) .
$$

One can check that $\mathbb{E}\left[X(x) X\left(x^{\prime}\right)\right]=\pi \int_{0}^{\infty} p_{D}\left(s, x, x^{\prime}\right) d s=G_{D}\left(x, x^{\prime}\right)$. The corresponding cut-off approximations are given by:

$$
X_{t}(x)=\sqrt{\pi} \int_{D \times\left[e^{-2 t}, \infty[\right.} p_{D}\left(\frac{s}{2}, x, y\right) W(d y, d s) .
$$

One can then easily show that:

$$
M_{t}^{\prime}(d x)=\left(2 \mathbb{E}\left[X_{t}(x)^{2}\right]-X_{t}(x)\right) e^{2 X_{t}(x)-2 \mathbb{E}\left[X_{t}(x)^{2}\right]} d x .
$$

converges to a non trivial positive and atomless random measure $M^{\prime}$. One can also show that:

$$
\sqrt{t} M_{t}(d x) \rightarrow \sqrt{\frac{2}{\pi}} M^{\prime}(d x), \quad \text { in probability as } t \rightarrow \infty .
$$

and that all the other results of this paper apply to $M^{\prime}$ (KPZ, existence of moments, etc...).

Let us nevertheless stress two points. Firstly, this approach is one way of constructing the critical Liouville measure but it does not show that all the other approaches yield the same measure. One must then extend the theories developped in $[34,54,55]$ or the one developped in [20] to show that there is a unique critical measure, i.e. independent of the limiting procedure. Secondly, by analogy with the star scale invariant case, we believe 
that the (properly shifted and normalized) law of the maximum of the GFF on a discrete lattice with mesh going to 0 converges in law to the sum of $\ln M^{\prime}(D)$ and an independent Gumbel variable (up to some constant $c$; see our conjecture 12 in [17]). Indeed, this should be the case if the discrete lattice is triangular as the discrete GFF on a triangular grid is a projection of the continuous GFF on the functions which are affine in the triangles (see [56]). There is no reason to believe that the form of the grid plays any specific role, at least if the lattice is regular enough. This gives in particular an explicit candidate to the limit law of [11].

\subsection{Remarks about atomic measures at criticality}

In dimension two, the Liouville quantum gravity measure on a domain $D$ is sometimes interpreted as the image of the intrinsic measure of a random surface $\mathcal{M}$ under a conformal map that sends $\mathcal{M}$ to $D$. This type of "surface" is highly singular (not a manifold in the usual sense). In certain limiting cases where the surface develops singular "bottlenecks", one expects the image measure on $D$ to become an atomic measure. In a certain sense (that we will not explain here), constructing these atomic measures requires one to replace $\gamma<2$ by a "dual value" $\gamma^{\prime}>2$ satisfying $\gamma \gamma^{\prime}=4$.

It is interesting to consider the analogous atomic measure in the critical case $\gamma=\gamma^{\prime}=2$ and to think about what its physical significance might be. We believe that the both the $\gamma=2$ measures (treated in this paper) and their $\gamma^{\prime}=2$ "atomic measure" variants (see below) have been studied in the physics literature before. However, when reading the physics literature about $\gamma=\gamma^{\prime}=2$ Liouville quantum gravity, it is sometimes difficult to sort out which physical constructions correspond to which mathematical objects. The remainder of this subsection will describe some of the history of these constructions and their relationship to the current work. This discussion can be safely skipped by the reader without specific background or interest in this area.

The issue of mathematically constructing singular Liouville measures beyond the phase transition, namely for $\gamma^{2}>4$, and deriving the corresponding (non-standard dual) KPZ formula has been investigated in $[6,18,21]$, giving the first mathematical understanding of the so-called duality in Liouville quantum gravity (see [3, 4, 13, 19, 23, 33, 36, 37, 38, 42] for an account of physics motivations). It thus remains to complete the mathematical Liouville quantum gravity picture at criticality, i.e. for $\gamma^{2}=4$. From the physics perspective, Liouville quantum gravity at criticality has been investigated in $[12,28,29,30$, $31,35,37,40,41,43,49,50,58]$. The reader is also referred to [17] for a brief summary about the physics literature on Liouville quantum gravity at criticality. Let us just stress that the critical case $\gamma=2$ corresponds to the value $c=1$ of the so-called central charge $c$ of the conformal field theory coupled to gravity, via the famous KPZ result [39]:

$$
\gamma=(\sqrt{25-c}-\sqrt{1-c}) / \sqrt{6}, \quad c \leqslant 1 .
$$

The Liouville measure at criticality presents an unusual dependence on the Liouville field $\varphi$ (equivalent to $X$ here) of the so-called "tachyon field" $T(\varphi) \propto \varphi e^{2 \varphi}[35,37,50]$. Its integral over a "background" Borelian set $A$ generates the quantum area $\mathcal{A}=\int_{A} T(\varphi) d x$, 
that we can recognize as the formal heuristic expression for the derivative measure (4). The possibility at criticality of another tachyon field of the atypical form $T(\varphi) \propto e^{2 \varphi}$ nevertheless appears in $[31,35,38]$. This form seems to heuristically correspond to a measure of type (1) (which actually vanishes for $\gamma=2$ ). At first sight, our result (5) here then seems to suggest that, up to the requested renormalization (5) of (1), the atypical tachyon field would actually coincide with the usual $\varphi e^{2 \varphi}$ tachyon field.

However, this atypical tachyon field $e^{2 \varphi}$ in Liouville quantum gravity has been associated to another, non-standard, form of the critical $c=1, \gamma=2$ random surface models. Indeed, the introduction of higher trace terms in the action of the $c=1$ matrix model of two-dimensional quantum gravity is known to generate a new critical behavior of the random surface [31, 35, 37, 58], with an enhanced critical proliferation of spherical bubbles connected one to another by microscopic "wormholes".

In order to model this non-standard critical theory, it might be necessary to modify the measures introduced here by explicitly introducing "atoms" on top of them, using the approach of $[6,18,21]$ for adding atoms to $\gamma<2$ random measures $M_{\gamma}$ in the description of the dual phase of Liouville quantum gravity. The "dual Liouville measure" corresponding to $\gamma<2$ involves choosing a Poisson point process from $\eta^{-\alpha-1} d \eta M_{\gamma}(d x)$, where $\alpha=\gamma^{2} / 4 \in(0,1)$, and letting each point $(\eta, x)$ in this process indicate an atom of size $\eta$ at location $x$. When $\gamma=2$ and $\alpha=1$, we can replace $M_{\gamma}$ with the derivative measure $M^{\prime}$ (4) (i.e., the limit (5)), and use the same construction; in this case (since $\alpha=1)$ the measure a.s. assigns infinite mass to each positive-Lebesgue-measure set $A \in$ $\mathcal{B}\left(\mathbb{R}^{d}\right)$. It is nonetheless still well-defined as a measure, and all of its (infinite) mass resides on a countable collection of atoms, each with finite mass. Alternatively, one may use standard Lévy compensation (intuitively, this amounts to replacing an "infinite measure" with an "infinite measure minus its expectation", interpreted in such a way that the result is finite) to produce a random distribution whose integral against any smooth test function is a.s. a finite (signed) value. One may expect that this construction yields the continuum random measure associated with the non-standard $c=1, \gamma=2$ Liouville random surface with enhanced bottlenecks, as described in [31, 35, 58], thus giving a mathematical interpretation to the (formal) tachyon field $e^{2 \varphi}$ that differs from the renormalized measure (5).

\section{Setup}

\subsection{Notations}

For a Borelian set $A \subset \mathbb{R}^{d}, \mathcal{B}(A)$ stands for the Borelian sigma-algebra on $A$. All the considered fields are constructed on the same probability space $(\Omega, \mathcal{F}, \mathbb{P})$. We denote by $\mathbb{E}$ the corresponding expectation. Given a Borelian set $A \subset \mathbb{R}^{d}$, we denote by $A^{c}$ its complement in $\mathbb{R}^{d}$. The relation $f \asymp g$ means that there exists a positive constant $c>0$ such that $c^{-1} f(x) \leqslant g(x) \leqslant c f(x)$ for all $x$. 


\section{$2.2 \star$-scale invariant kernels}

Here we introduce the Gaussian fields that we will use throughout the paper. We consider a family of centered stationary Gaussian processes $\left(\left(X_{t}(x)\right)_{x \in \mathbb{R}^{d}}\right)_{t \geqslant 0}$ where, for each $t \geqslant 0$, the process $\left(X_{t}(x)\right)_{x \in \mathbb{R}^{d}}$ has covariance given by:

$$
K_{t}(x)=\mathbb{E}\left[X_{t}(0) X_{t}(x)\right]=\int_{1}^{e^{t}} \frac{k(u x)}{u} d u
$$

for some covariance kernel $k$ satisfying $k(0)=1$, of class $C^{1}$ and vanishing outside a compact set (actually this latter condition is not necessary but it simplifies the presentation). We also assume that the process $\left(X_{t}(x)-X_{s}(x)\right)_{x \in \mathbb{R}^{d}}$ is independent of the processes $\left(\left(X_{u}(x)\right)_{x \in \mathbb{R}^{d}}\right)_{u \leqslant s}$ for all $s<t$. In other words, the mapping $t \mapsto X_{t}(\cdot)$ has independent increments. Such a construction of Gaussian processes is carried out in [2]. For $\gamma \geqslant 0$, we consider the approximate Gaussian multiplicative chaos $M_{t}^{\gamma}(d x)$ on $\mathbb{R}^{d}$ :

$$
M_{t}^{\gamma}(d x)=e^{\gamma X_{t}(x)-\frac{\gamma^{2}}{2} E\left[X_{t}(x)^{2}\right]} d x
$$

It is well known $[2,34]$ that, almost surely, the family of random measures $\left(M_{t}^{\gamma}\right)_{t>0}$ weakly converges as $t \rightarrow \infty$ towards a random measures $M^{\gamma}$, which is non-trivial for $\gamma^{2}<2 d$. The purpose of this paper is to investigate the phase transition, that is $\gamma^{2}=2 d$. Remind that we have $[17,34]$ :

Proposition 1. For $\gamma^{2}=2 d$ (and also for $\gamma^{2}>2 d$ ), the standard construction (7) yields a vanishing limiting measure:

$$
\lim _{t \rightarrow \infty} M_{t}^{\gamma}(d x)=0 \quad \text { almost surely }
$$

One of the main purposes of this article is to give a non trivial renormalization of the family $\left(M_{t}^{\sqrt{2 d}}\right)_{t}$. We stress that a suitable renormalization should yield a non trivial solution to the lognormal star-equation:

Definition 2. Log-normal $\star$-scale invariance. A random Radon measure $M$ is said to be lognormal $\star$-scale invariant if for all $0<\varepsilon \leqslant 1, M$ obeys the cascading rule

$$
\left.(M(A))_{A \in \mathcal{B}\left(\mathbb{R}^{d}\right)} \stackrel{\text { law }}{=}\left(\int_{A} e^{\omega_{\varepsilon}(r)} M^{\varepsilon}(d r)\right)\right)_{A \in \mathcal{B}\left(\mathbb{R}^{d}\right)}
$$

where $\omega_{\varepsilon}$ is a stationary stochastically continuous Gaussian process and $M^{\varepsilon}$ is a random measure independent from $\omega_{\varepsilon}$ satisfying the scaling relation

$$
\left(M^{\varepsilon}(A)\right)_{A \in \mathcal{B}\left(\mathbb{R}^{d}\right)} \stackrel{\text { law }}{=}\left(M\left(\frac{A}{\varepsilon}\right)\right)_{A \in \mathcal{B}\left(\mathbb{R}^{d}\right)} .
$$


Let us mention that the authors in [2] have proved that, for $\gamma^{2}<2 d$, the measure $M^{\gamma}$ is lognormal $\star$-scale invariant with

$$
\omega_{\varepsilon}(r)=\gamma X_{\ln \frac{1}{\varepsilon}}(r)-\left(\frac{\gamma^{2}}{2}+d\right) \ln \frac{1}{\varepsilon},
$$

where $X_{\ln \frac{1}{\varepsilon}}$ is the Gaussian process introduced in (6). Furthermore this scaling relation still makes perfect sense when the scaling factor $\omega_{\varepsilon}$ is given by (11) for the value $\gamma^{2}=2 d$. Therefore, to define a natural Gaussian multiplicative chaos at the value $\gamma^{2}=2 d$, one has to look for a solution to this equation when the scaling factor is given by (11) with $\gamma^{2}=2 d$ and conversely, each random measure candidate for being a Gaussian multiplicative chaos at the value $\gamma^{2}=2 d$ must satisfy these relations. In [17], a non trivial solution has been constructed, called the derivative martingale. Since it is conjectured that all the non trivial ergodic solutions to this equation (actually we also need to impose a sufficient decay of the covariance kernel of the process $\omega_{\varepsilon}$, see [2] for further details) are equal up to a multiplicative factor, it is expected that a non trivial renormalization of the family $\left(M_{t}^{\sqrt{2 d}}\right)_{t}$ converges towards the derivative martingale. Proving this is the first purpose of this paper. The second purpose is to prove that the derivative martingale satisfies the KPZ formula.

The organization of this paper is as follows. In Section 3, we introduce the derivative martingale and remind the reader of its properties as stated in [17]. In Section 4, we explain how to renormalize $\left(M_{t}^{\sqrt{2 d}}\right)_{t}$ to obtain the derivative martingale. This entails many non trivial moment estimates for the derivative martingale. In Section 5, we prove that these moment estimates allow to obtain the KPZ formula at criticality.

Remark 3. As observed in [17], we stress that the main motivation for considering $\star-$ scale invariant kernels is the connection between the associated random measures and the $\star$-equation. Nevertheless, our proofs can be easily generalized. First, we stress that the assumption about the compact support of $k$ involved in (6) may be relaxed, provided that one imposes some restrictions about the decay of $k$ at infinity. Generally speaking, these restrictions are rather weak but may require some tedious extra computations. For instance, it is not very difficult to see that the case when $k$ is $C^{1}$ with an exponential decay for $k$ and $\nabla k$ does work. One may also wonder about the case of more general Gaussian multiplicative chaos of log-correlated Gaussian fields "à la Kahane" [34]. It is not difficult to see that these other chaos can be written as in (6), with a kernel $k$ depending also on the scale $u$. Then the same restrictions about the decay of $k(u, \cdot)$ at infinity apply. Let us just add that one can carry out the renormalization approach associated to exact scale invariant kernels as constructed in [5, 52].

\section{Derivative martingale}

A way of constructing a solution to the $\star$-equation at the critical value $\gamma^{2}=2 d$ is to introduce the derivative martingale $M_{t}^{\prime}(d x)$ defined by:

$$
M_{t}^{\prime}(d x)=\left(\sqrt{2 d} t-X_{t}(x)\right) e^{\sqrt{2 d} X_{t}(x)-d \mathbb{E}\left[X_{t}(x)^{2}\right]} d x .
$$


It is plain to see that, for each open bounded set $A \subset \mathbb{R}^{d}$, the family $\left(M_{t}^{\prime}(A)\right)_{t}$ is a martingale. Nevertheless, it is not nonnegative. It is therefore not obvious that such a family converges towards a (non trivial) positive limiting random variable. The following theorem has been proved in [17]:

Theorem 4. For each bounded open set $A \subset \mathbb{R}^{d}$, the martingale $\left(M_{t}^{\prime}(A)\right)_{t \geqslant 0}$ converges almost surely towards a positive random variable denoted by $M^{\prime}(A)$, such that $M^{\prime}(A)>0$ almost surely. Consequently, almost surely, the (locally signed) random measures $\left(M_{t}^{\prime}(d x)\right)_{t \geqslant 0}$ converge weakly as $t \rightarrow \infty$ towards a positive random measure $M^{\prime}(d x)$. This limiting measure has full support and is atomless. Furthermore, the measure $M^{\prime}$ is a solution to the $\star$-equation (9) with $\gamma=\sqrt{2 d}$.

\section{Renormalization}

The main purpose of this paper is to establish that the derivative martingale can be seen as the limit of a suitable renormalization of the family $\left(M_{t}^{\sqrt{2 d}}\right)_{t}$.

Theorem 5. The family $\left(\sqrt{t} M_{t}^{\sqrt{2 d}}\right)_{t}$ converges in probability as $t \rightarrow \infty$ towards a non trivial limit, which turns out to be the same, up to a multiplicative constant, as the limit of the derivative martingale. More precisely, we have for all bounded open set A:

$$
\sqrt{t} M_{t}^{\sqrt{2 d}}(A) \rightarrow \sqrt{\frac{2}{\pi}} M^{\prime}(A), \quad \text { in probability as } t \rightarrow \infty .
$$

The main advantage of this renormalization approach is to make the derivative martingale appear as a limit of integrals over exponentials of the field: this is useful to use Kahane's convexity inequality (see (10)). We can then prove:

Corollary 6. The positive random measure $M^{\prime}(d x)$ possesses moments of order $q$ for all $q<1$. Furthermore, for all $q>0$ and every non-empty bounded open set $A$, we have

$$
\sup _{t \geqslant 1} \mathbb{E}\left[\left(\frac{1}{\sqrt{t} M_{t}^{\sqrt{2 d}}(A)}\right)^{q}\right]<+\infty .
$$

We can then determine the power law spectrum of the random measure $M^{\prime}$ :

Corollary 7. The power law spectrum of the random measure $M^{\prime}$ is given for $0 \leqslant q<1$ by

$$
\xi(q)=2 d q-d q^{2} .
$$

More precisely, for each bounded open set $A$ of $\mathbb{R}^{d}$, we have

$$
\forall q<1, \quad \mathbb{E}\left[M^{\prime}(\lambda A)^{q}\right] \asymp C_{q} \lambda^{\xi(q)}
$$

when $\lambda$ goes to 0 , and where the coefficient $C_{q}$ depends on $q$. 


\section{KPZ formula}

\subsection{The KPZ formula}

In this section, we investigate the KPZ formula for the derivative martingale, which corresponds to the natural construction of Gaussian multiplicative chaos at criticality $\gamma^{2}=2 d$. The KPZ formula is a relation between the Hausdorff dimensions of a given set $A$ as measured by the Lebesgue measure or $M^{\prime}$. So we first recall how to define these dimensions. Given an atomless Radon measure $\mu$ on $\mathbb{R}^{d}$ and $s \in[0,1]$, we define

$$
H_{\mu}^{s, \delta}(A)=\inf \left\{\sum_{k} \mu\left(B_{k}\right)^{s}\right\}
$$

where the infimum runs over all the covering $\left(B_{k}\right)_{k}$ of $A$ with open Euclidean balls centered at $A$ with radius $r_{k} \leqslant \delta$. Clearly, the mapping $\delta>0 \mapsto H_{\mu}^{s, \delta}(A)$ is decreasing. Hence we can define the s-dimensional $\mu$-Hausdorff outer measure:

$$
H_{\mu}^{s}(A)=\lim _{\delta \rightarrow 0} H_{\mu}^{s, \delta}(A) .
$$

The limit exists but may be infinite. $H_{\mu}^{s}$ is a metric outer measure on $\mathbb{R}^{d}$ (see [25] for definitions). Thus $H_{\mu}^{s}$ is a measure on the $\sigma$-field of $H_{\mu}^{s}$-measurable sets, which contains all the Borelian sets.

The $\mu$-Hausdorff dimension of the set $A$ is then defined as the value

$$
\operatorname{dim}_{\mu}(A)=\inf \left\{s \geqslant 0 ; H_{\mu}^{s}(A)=0\right\} .
$$

Notice that $\operatorname{dim}_{\mu}(A) \in[0,1]$. Since $\mu$ is atomless, the Hausdorff dimension is also characterized by:

$$
\operatorname{dim}_{\mu}(A)=\sup \left\{s \geqslant 0 ; H_{\mu}^{s}(A)=+\infty\right\} .
$$

Since $M^{\prime}$ does not possess atoms, this relation is valid for $M^{\prime}$. This allows to characterize the Hausdorff dimension as the threshold value at which the mapping $s \mapsto H_{\mu}^{s}(A)$ jumps from $+\infty$ to 0 .

In what follows, given a compact set $K$ of $\mathbb{R}^{d}$, we define its Hausdorff dimensions $\operatorname{dim}_{L e b}(K)$ and $\operatorname{dim}_{M^{\prime}}(K)$ computed as indicated above with $\mu$ respectively equal to the Lebesgue measure or $M^{\prime}$. So, a priori, the value of $\operatorname{dim}_{M^{\prime}}(K)$ is random. Nevertheless, a straightforward $0-1$ law argument shows that $\operatorname{dim}_{M^{\prime}}(K)$ is actually deterministic. We reinforce this intuition by stating:

Theorem 8 . KPZ at criticality $\gamma^{2}=\mathbf{2 d}$. Let $K$ be a compact set of $\mathbb{R}^{d}$. Almost surely, we have the relation

$$
\operatorname{dim}_{L e b}(K)=\frac{\xi\left(\operatorname{dim}_{M^{\prime}}(K)\right)}{d}=2 d \operatorname{dim}_{M^{\prime}}(K)-d \operatorname{dim}_{M^{\prime}}(K)^{2} .
$$

Remark 9. Let us stress that our proof also allows one to choose $K$ random but independent of the measure $M$. We could also consider sets $K$ depending on $M$ through the first scales of $M$, that is depending on $\left(X_{s}(x)\right)_{x \in \mathbb{R}^{d}, s \leqslant T}$ for some $T>0$. Strengthening this dependence would give rise to non trivial additional difficulties. 


\subsection{Heuristics and open questions on the KPZ formula}

Here, we give a direct and heuristic derivation of Theorem 8 for the following reasons:

- it gives a quick intuitive idea of why Theorem 8 is valid;

- it enlightens the idea behind the proof of Theorem 8 (which involves introducing very particular Frostman measures);

- it leads in a natural way to open questions which can be seen as generalizations (or complements to) Theorem 8.

In fact, we will work in the subcritical case $\gamma^{2}<2 d$ (a similar heuristic can be derived for the case $\gamma^{2}=2 d$ ). Recall that lognormal $\star$-scale invariance for $M$, defined by (1) with $\gamma^{2}<2 d$, amounts to the following equivalent:

$$
M(B(x, r)) \sim r^{d} e^{\gamma X_{\ln \frac{1}{r}}(x)-\frac{\gamma^{2}}{2} \ln \frac{1}{r}}
$$

where $\sim$ denotes that both quantities are equal up to multiplication by a random factor of order 1 which does not depend on $r$ (note that the random factor depends on $x$ ). If we set $\xi_{\gamma}(s)=\left(d+\frac{\gamma^{2}}{2}\right) s-\frac{\gamma^{2}}{2} s^{2}$, it is thus tempting to write the following equivalents for a set $K$ :

$$
\begin{aligned}
H_{M}^{s}(K) & =\lim _{\delta \rightarrow 0} \inf \left\{\sum_{k} M\left(B\left(x_{k}, r_{k}\right)\right)^{s}, K \subset \cup_{k} B\left(x_{k}, r_{k}\right),\left|r_{k}\right| \leqslant \delta\right\} \\
& \sim \lim _{\delta \rightarrow 0} \inf \left\{\sum_{k}\left(r_{k}^{d} e^{\gamma X_{\ln \frac{1}{r_{k}}}\left(x_{k}\right)-\frac{\gamma^{2}}{2} \ln \frac{1}{r_{k}}}\right)^{s}, K \subset \cup_{k} B\left(x_{k}, r_{k}\right),\left|r_{k}\right| \leqslant \delta\right\} \\
& =\lim _{\delta \rightarrow 0} \inf \left\{\sum_{k} e^{s \gamma X_{\ln \frac{1}{r_{k}}}\left(x_{k}\right)-\frac{s^{2} \gamma^{2}}{2} \ln \frac{1}{r_{k}}} r_{k}^{\xi_{\gamma}(s)}, K \subset \cup_{k} B\left(x_{k}, r_{k}\right),\left|r_{k}\right| \leqslant \delta\right\} \\
& \sim \int_{K} e^{s \gamma X(x)-\frac{s^{2} \gamma^{2}}{2} \mathbb{E}\left[X(x)^{2}\right]} H^{\xi_{\gamma}(s) / d}(d x),
\end{aligned}
$$

where the last term is a Gaussian multiplicative chaos applied to the Radon measure $H^{\xi_{\gamma}(s) / d}(K \cap d x)$ (at least if $\left.H^{\xi_{\gamma}(s) / d}(K)<\infty\right)$. This heuristics shows that the quantum Hausdorff measure $H_{M}^{s}$ should not be too far from $\int e^{s \gamma X(x)-\frac{s^{2} \gamma^{2}}{2} \mathbb{E}\left[X(x)^{2}\right]} H^{\xi_{\gamma}(s) / d}(d x)$ (though up to possible logarithmic corrections). In particular, $H_{M}^{s}(K)$ is of order 1 if and only if $H^{\xi_{\gamma}(s) / d}(K)$ is of order 1 . Note that the $e^{s \gamma X(x)-\frac{s^{2} \gamma^{2}}{2} \mathbb{E}\left[X(x)^{2}\right]} H^{\xi_{\gamma}(s) / d}(d x)$ measure appears in the physics litterature on KPZ in the so-called "gravitational dressing" (see, e.g., [27]), and in the rigorous context of the coupling of Schramm-Loewner Evolution to Liouville quantum gravity [22]. Naturally, one could ask to what extent the above heuristics can be made rigorous. 


\section{A. Auxiliary results}

We first state the classical "Kahane's convexity inequalities" (originally written in [34] see also [2, 55] for a proof in English):

Lemma 10. Let $F: \mathbb{R}_{+} \rightarrow \mathbb{R}$ be some convex function such that

$$
\forall x \in \mathbb{R}_{+}, \quad|F(x)| \leqslant M\left(1+|x|^{\beta}\right),
$$

for some positive constants $M, \beta$, and $\sigma$ be a Radon measure on the Borelian subsets of $\mathbb{R}^{d}$. Given a bounded Borelian set $A$, let $\left(X_{r}\right)_{r \in A},\left(Y_{r}\right)_{r \in A}$ be two continuous centered Gaussian processes with continuous covariance kernels $k_{X}$ and $k_{Y}$ such that

$$
\forall u, v \in A, \quad k_{X}(u, v) \leqslant k_{Y}(u, v)
$$

Then

$$
\mathbb{E}\left[F\left(\int_{A} e^{X_{r}-\frac{1}{2} \mathbb{E}\left[X_{r}^{2}\right]} \sigma(d r)\right)\right] \leqslant \mathbb{E}\left[F\left(\int_{A} e^{Y_{r}-\frac{1}{2} \mathbb{E}\left[Y_{r}^{2}\right]} \sigma(d r)\right)\right] .
$$

If we further assume

$$
\forall u \in A, \quad k_{X}(u, u)=k_{Y}(u, u)
$$

then for any increasing function $F: \mathbb{R}_{+} \rightarrow \mathbb{R}$ :

$$
\mathbb{E}\left[F\left(\sup _{x \in A} Y_{x}\right)\right] \leqslant \mathbb{E}\left[F\left(\sup _{x \in A} X_{x}\right)\right]
$$

\section{B. Proofs of Section 4}

We denote by $\mathcal{F}_{t}$ the sigma algebra generated by $\left\{X_{s}(x) ; s \leqslant t, x \in \mathbb{R}^{d}\right\}$ and by $\mathcal{F}$ the sigma algebra generated by $\bigcup_{t} \mathcal{F}_{t}$. Given a fixed open bounded set $A \subset \mathbb{R}^{d}$ and parameters $t, \beta>0$, we introduce the random variables

$$
\begin{aligned}
& Z_{t}^{\beta}(A)=\int_{A}\left(\sqrt{2 d} t-X_{t}(x)+\beta\right) \mathbb{I}_{\left\{\tau_{x}^{\beta}>t\right\}} e^{\sqrt{2 d} X_{t}(x)-d t} d x \\
& R_{t}^{\beta}(A)=\int_{A} \mathbb{I}_{\left\{\tau_{x}^{\beta}>t\right\}} e^{\sqrt{2 d} X_{t}(x)-d t} d x
\end{aligned}
$$

where, for each $x \in A, \tau_{x}^{\beta}$ is the stopping time adapted to the filtration $\mathcal{G}_{t}=\sigma\left(X_{s}(x) ; s \leqslant t, x \in\right.$ $\mathbb{R}^{d}$ ) defined by

$$
\tau_{x}^{\beta}=\inf \left\{u>0, X_{u}(x)-\sqrt{2 d} u>\beta\right\}
$$

For $x \in \mathbb{R}^{d}$, we also define

$$
f_{t}^{\beta}(x)=\left(\sqrt{2 d} t-X_{t}(x)+\beta\right) \mathbb{I}_{\left\{\tau_{x}^{\beta}>t\right\}} e^{\sqrt{2 d} X_{t}(x)-d t} .
$$

It is plain to check that for each $\beta>0$ and each bounded open set $A,\left(Z_{t}^{\beta}(A)\right)_{t}$ is a nonnegative martingale with respect to $\left(\mathcal{F}_{t}\right)_{t}$ such that $\mathbb{E}\left[Z_{t}^{\beta}(A)\right]=\beta|A|$. It is proved in 
[17] that it is uniformly integrable and therefore almost surely converges towards a non trivial limit.

We first stress that, for each $x$ fixed, the process $t \mapsto X_{t}(x)$ is standard Brownian motion. We will repeatedly use this fact throughout the proof without mentioning it again.

\section{B.1 Rooted measure}

Since for each $x,\left(f_{t}^{\beta}(x)\right)_{t}$ is a martingale, we can define the probability measure $\Theta_{t}^{\beta}$ on $\mathcal{B}(A) \otimes \mathcal{F}_{t}$ by

$$
\Theta_{t}^{\beta}=\frac{f_{t}^{\beta}(x)}{\beta|A|} d x d \mathbb{P} .
$$

We denote by $\mathbb{E}_{\Theta_{t}^{\beta}}$ the corresponding expectation. In fact, since the above definition defines a pre-measure on the ring $\bigcup_{t} \mathcal{F}_{t}$, one can define the rooted measure $\Theta^{\beta}$ on $\mathcal{B}(A) \otimes \mathcal{F}$ by using Caratheodory's extension theorem. We recover $\Theta_{\mid \mathcal{B}(A) \otimes \mathcal{F}_{t}}^{\beta}=\Theta_{t}^{\beta}$. We observe that $\Theta_{t}^{\beta}\left(Z_{t}^{\beta}(A)>0\right)=1$ for any $t$.

Similarly, we construct the probability measure $Q^{\beta}$ on $\mathcal{F}$ by setting:

$$
Q_{\mid \mathcal{F}_{t}}^{\beta}=\frac{Z_{t}^{\beta}(A)}{\beta|A|} d \mathbb{P}
$$

which is nothing but the marginal law of $(\omega, x) \mapsto \omega$ with respect to $\Theta_{t}^{\beta}$. Since $\left(Z_{t}^{\beta}(A)\right)_{t \geqslant 0}$ is a uniformly integrable martingale which converges to a limit $Z^{\beta}(A)$, we can also define $Q^{\beta}$ directly on $\mathcal{F}$ by:

$$
Q^{\beta}=\frac{Z^{\beta}(A)}{\beta|A|} d \mathbb{P} .
$$

We state a few elementary results below. The conditional law of $x$ given $\mathcal{F}_{t}$ is given by:

$$
\Theta_{t}^{\beta}\left(d x \mid \mathcal{F}_{t}\right)=\frac{f_{t}(x)}{Z_{t}^{\beta}(A)} d x .
$$

If $Y$ is a $\mathcal{B}(A) \otimes \mathcal{F}_{t}$-measurable random variable then it has the following conditional expectation given $\mathcal{F}_{t}$ :

$$
\mathbb{E}_{\Theta_{t}^{\beta}}\left[Y \mid \mathcal{F}_{t}\right]=\int_{A} Y(x, \omega) \frac{f_{t}^{\beta}(x)}{Z_{t}^{\beta}(A)} d x .
$$

In particular, for any event $E \in \mathcal{F}_{t}$, we have

$$
\mathbb{E}_{Q^{\beta}}\left[\mathbb{1}_{E} \mathbb{E}_{\Theta_{t}^{\beta}}\left[Y \mid \mathcal{F}_{t}\right]\right]=\mathbb{E}_{Q^{\beta}}\left[\mathbb{1}_{E} \int_{A} Y(x, \omega) \frac{f_{t}^{\beta}(x)}{Z_{t}^{\beta}(A)} d x\right]=\mathbb{E}_{\Theta_{t}^{\beta}}\left[\mathbb{I}_{E} Y\right] .
$$

Under $\Theta_{t}^{\beta}$, the law of the random process $\left(\beta+\sqrt{2 d} s-X_{s}\right)_{s} \leqslant t$ is that of a 3 -dimensional Bessel process started at $\beta$. In what follows, we will use the notation:

$$
Y_{s}^{\beta}(x)=\beta+\sqrt{2 d} s-X_{s}(x) .
$$


Of course, $Y_{s}^{0}(x)$ simply stands for $\sqrt{2 d} s-X_{s}(x)$.

The proof is inspired from [1]. Mainly, we follow their argument. Nevertheless, we make two remarks. First, most of the auxiliary estimates obtained in [1] about the minimum of the underlying random walk are much easier to obtain in our context because of the Gaussian nature of our framework (in particular, the random walk conditioned to stay positive is here a Bessel process). Second, the continuous structure makes correlations much more intricate to get rid of: we have no spinal decomposition at our disposal, no underlying tree structure, etc. We adapt some arguments developed in [17] at this level.

\section{B.2 Proofs under the rooted measure}

The first step is to prove the convergence under $Q^{\beta}$. This subsection is thus entirely devoted to the proof of the following result:

Proposition 11. Given $\beta>0$, we have

$$
\mathbb{E}_{Q^{\beta}}\left[\frac{R_{t}^{\beta}(A)}{Z_{t}^{\beta}(A)}\right] \simeq \sqrt{\frac{2}{\pi t}} \quad \text { as } t \rightarrow \infty
$$

and

$$
\mathbb{E}_{Q^{\beta}}\left[\left(\frac{R_{t}^{\beta}(A)}{Z_{t}^{\beta}(A)}\right)^{2}\right] \simeq \frac{2}{\pi t} \quad \text { as } t \rightarrow \infty .
$$

As a consequence, under $Q^{\beta}$, we have

$$
\lim _{t \rightarrow \infty} \sqrt{t} \frac{R_{t}^{\beta}(A)}{Z_{t}^{\beta}(A)}=\sqrt{\frac{2}{\pi}} \quad \text { in probability. }
$$

Proof. It is clear that $(17)+(18)$ imply that the variance of the ratio $\frac{\sqrt{t} R_{t}^{\beta}(A)}{Z_{t}^{\beta}(A)}$ under $Q^{\beta}$ goes to 0 a $t$ goes to $\infty$. Therefore, under $Q^{\beta}$, this ratio converges in quadratic mean towards $\sqrt{\frac{2}{\pi}}$, and hence in probability, thus proving (19).

It is plain to establish the relation (17). First observe that:

$$
\begin{aligned}
\mathbb{E}_{Q^{\beta}}\left[\frac{R_{t}^{\beta}(A)}{Z_{t}^{\beta}(A)}\right] & =\frac{1}{\beta|A|} \mathbb{E}\left[R_{t}^{\beta}(A)\right] \\
& =\frac{1}{\beta} \mathbb{E}\left[\mathbb{I}_{\left\{\tau_{x}^{\beta}>t\right\}} e^{\sqrt{2 d} X_{t}(x)-d t}\right]
\end{aligned}
$$

Let us denote by $B$ a standard one-dimensional Brownian motion. By using the Girsanov 
transform, we have:

$$
\begin{aligned}
\mathbb{E}_{Q^{\beta}}\left[\frac{R_{t}^{\beta}(A)}{Z_{t}^{\beta}(A)}\right] & =\frac{1}{\beta} \mathbb{P}\left(\sup _{0 \leqslant u \leqslant t} B_{u} \leqslant \beta\right) \\
& =\frac{1}{\beta} \mathbb{P}\left(\left|B_{t}\right| \leqslant \beta\right) \\
& \simeq \sqrt{\frac{2}{\pi t}} \quad \text { as } t \rightarrow \infty .
\end{aligned}
$$

Relation (17) is established.

The proof of (18) is much more involved and will be carried out in several steps. To begin with, it may be worth sketching the strategy of the proof:

1. Observe that

$$
\frac{R_{t}^{\beta}(A)}{Z_{t}^{\beta}(A)}=\mathbb{E}_{\Theta_{t}^{\beta}}\left[\frac{1}{Y_{t}^{\beta}(x)} \mid \mathcal{F}_{t}\right]
$$

2. Deduce that

$$
\mathbb{E}_{Q^{\beta}}\left[\left(\frac{R_{t}^{\beta}(A)}{Z_{t}^{\beta}(A)}\right)^{2}\right]=\mathbb{E}_{\Theta_{t}^{\beta}}\left[\frac{R_{t}^{\beta}(A)}{Z_{t}^{\beta}(A)} \times \frac{1}{Y_{t}^{\beta}(x)}\right] .
$$

3. The third step consists in subtracting a ball centered at $x$ with radius $e^{-h_{t}}$ to the set $A$, call it $B\left(x, e^{-h_{t}}\right)$. This is convenient because if the radius is well chosen the ratio $\frac{R_{t}^{\beta}\left(A \backslash B\left(x, e^{-h_{t}}\right)\right)}{Z_{t}^{\beta}\left(A \backslash B\left(x, e^{-h_{t}}\right)\right)}$ and the weight $\frac{1}{Y_{t}^{\beta}(x)}$ will be "almost" independent. Roughly speaking, the reason why we can subtract a ball is that the measure does not possess atom. So, at least if the radius of the ball is small enough, it is always possible to subtract a ball centered at $x$ without radically affecting the behaviour of the quantity (21). In the forthcoming rigorous proof, we won't base our argument on the fact that the measure is atomless. Instead, we will use estimates on the process $Y_{t}^{\beta}(x)$ under the rooted measure, which amounts to the same (as proved in [17]).

4. The last step consists in factorizing (21) because of (almost) independence:

$$
\begin{aligned}
\mathbb{E}_{Q^{\beta}}\left[\left(\frac{R_{t}^{\beta}(A)}{Z_{t}^{\beta}(A)}\right)^{2}\right] & \simeq \mathbb{E}_{\Theta_{t}^{\beta}}\left[\frac{R_{t}^{\beta}\left(A \backslash B_{x, t}\right)}{Z_{t}^{\beta}\left(A \backslash B_{x, t}\right)} \times \frac{1}{Y_{t}^{\beta}(x)}\right] \\
& \simeq \mathbb{E}_{\Theta_{t}^{\beta}}\left[\frac{R_{t}^{\beta}\left(A \backslash B_{x, t}\right)}{Z_{t}^{\beta}\left(A \backslash B_{x, t}\right)}\right] \mathbb{E}_{\Theta_{t}^{\beta}}\left[\frac{1}{Y_{t}^{\beta}(x)}\right] \\
& \simeq \mathbb{E}_{Q^{\beta}}\left[\frac{R_{t}^{\beta}(A)}{Z_{t}^{\beta}(A)}\right] \mathbb{E}_{Q^{\beta}}\left[\frac{R_{t}^{\beta}(A)}{Z_{t}^{\beta}(A)}\right]
\end{aligned}
$$

This last quantity is equivalent for $t$ large to $\frac{2}{\pi t}$. Actually, most of the forthcoming computations are made to justify that the factorization can be made rigorously. This point is highly technical and the related computations may appear tedious to the reader. 
Now we begin with the rigorous proof of (18). Recall that we use the shorthand (16). Let us first claim:

Lemma 12. We have

$$
\mathbb{E}_{Q^{\beta}}\left[\left(\frac{R_{t}^{\beta}(A)}{Z_{t}^{\beta}(A)}\right)^{2}\right]=O\left(\frac{1}{t}\right) \quad \text { as } t \rightarrow \infty .
$$

Proof. By Jensen inequality, we have:

$$
\begin{aligned}
\mathbb{E}_{Q^{\beta}}\left[\left(\frac{R_{t}^{\beta}(A)}{Z_{t}^{\beta}(A)}\right)^{2}\right] & =\mathbb{E}_{Q^{\beta}}\left[\left(\mathbb{E}_{\Theta_{t}^{\beta}}\left[\frac{1}{Y_{t}^{\beta}(x)} \mid \mathcal{F}_{t}\right]\right)^{2}\right] \\
& \leqslant \mathbb{E}_{Q^{\beta}}\left[\mathbb{E}_{\Theta_{t}^{\beta}}\left[\frac{1}{Y_{t}^{\beta}(x)^{2}} \mid \mathcal{F}_{t}\right]\right] \\
& \leqslant \mathbb{E}_{\Theta_{t}^{\beta}}\left[\frac{1}{Y_{t}^{\beta}(x)^{2}}\right]
\end{aligned}
$$

Since, under $\Theta_{t}^{\beta}$, the law of the process $\left(Y_{s}^{\beta}(x)\right)_{s \leqslant t}$ is that of a 3-dimensional Bessel process starting at $\beta>0$, the lemma follows.

Now we will decompose the space in two parts: a part, call it $E_{t}$, where we have strong estimates on the process $\left(Y_{s}^{\beta}(x)\right)_{s \leqslant t}$ and an other part that we want to be "small". More precisely, let $E_{t}$ be a $\mathcal{B}(A) \otimes \mathcal{F}_{t}$-measurable event such that $\mathbb{E}_{\Theta_{t}^{\beta}}\left(\mathbb{I}_{E_{t}}\right) \rightarrow 1$ as $t$ goes to $\infty$. Let

$$
\xi_{t}=\mathbb{E}_{\Theta_{t}^{\beta}}\left[\mathbb{I}_{E_{t}^{c}} \frac{1}{Y_{t}^{\beta}(x)} \mid \mathcal{F}_{t}\right]
$$

Notice that

$$
\frac{R_{t}^{\beta}(A)}{Z_{t}^{\beta}(A)}=\mathbb{E}_{\Theta_{t}^{\beta}}\left[\frac{1}{Y_{t}^{\beta}(x)} \mid \mathcal{F}_{t}\right]=\xi_{t}+\mathbb{E}_{\Theta_{t}^{\beta}}\left[\mathbb{I}_{E_{t}} \frac{1}{Y_{t}^{\beta}(x)} \mid \mathcal{F}_{t}\right]
$$

We deduce:

$$
\mathbb{E}_{Q^{\beta}}\left[\left(\frac{R_{t}^{\beta}(A)}{Z_{t}^{\beta}(A)}\right)^{2}\right]=\mathbb{E}_{Q^{\beta}}\left[\frac{R_{t}^{\beta}(A)}{Z_{t}^{\beta}(A)} \xi_{t}\right]+\mathbb{E}_{\Theta_{t}^{\beta}}\left[\frac{R_{t}^{\beta}(A)}{Z_{t}^{\beta}(A)} \mathbb{I}_{E_{t}} \frac{1}{Y_{t}^{\beta}(x)}\right] .
$$

We will treat separately the two terms in the above right-hand side. The Cauchy-Schwarz inequality and Lemma 12 yield

$$
\mathbb{E}_{Q^{\beta}}\left[\frac{R_{t}^{\beta}(A)}{Z_{t}^{\beta}(A)} \xi_{t}\right] \leqslant \mathbb{E}_{Q^{\beta}}\left[\left(\frac{R_{t}^{\beta}(A)}{Z_{t}^{\beta}(A)}\right)^{2}\right]^{1 / 2} \mathbb{E}_{Q^{\beta}}\left[\xi_{t}^{2}\right]^{1 / 2} \leqslant \frac{C}{\sqrt{t}} \mathbb{E}_{Q^{\beta}}\left[\xi_{t}^{2}\right]^{1 / 2} .
$$

If we can prove that $\mathbb{E}_{Q^{\beta}}\left[\xi_{t}^{2}\right]=o\left(\frac{1}{t}\right)$ then $(24)$ will tell us that $\mathbb{E}_{Q^{\beta}}\left[\frac{R_{t}^{\beta}(A)}{Z_{t}^{\beta}(A)} \xi_{t}\right]=o\left(\frac{1}{t}\right)$. Therefore, Proposition 11, in particular (18), is a consequence of the two following lemma:

Lemma 13. Let $\beta>0$ and $E_{t}$ be an event such that $\mathbb{E}_{\Theta_{t}^{\beta}}\left(\mathbb{I}_{E_{t}}\right) \rightarrow 1$ as $t$ goes to $\infty$. We have

$$
\mathbb{E}_{Q^{\beta}}\left[\xi_{t}^{2}\right]=o\left(\frac{1}{t}\right)
$$


Lemma 14. Let $\beta>0$. There exists a family of events $E_{t}$ such that $\mathbb{E}_{\Theta_{t}^{\beta}}\left(\mathbb{I}_{E_{t}}\right) \rightarrow 1$ as $t$ goes to $\infty$ and

$$
\mathbb{E}_{\Theta_{t}^{\beta}}\left[\frac{R_{t}^{\beta}(A)}{Z_{t}^{\beta}(A)} \frac{\mathbb{1}_{E_{t}}}{Y_{t}^{\beta}(x)}\right] \leqslant \frac{2}{\pi t}+o\left(\frac{1}{t}\right)
$$

Proof of Lemma 13. Fix $\varepsilon>0$. By Jensen's inequality, we have

$$
\begin{aligned}
& \mathbb{E}_{Q^{\beta}}\left[\xi_{t}^{2}\right] \leqslant \mathbb{E}_{\Theta_{t}^{\beta}}\left[\mathbb{I}_{E_{t}^{c}} \frac{1}{Y_{t}^{\beta}(x)^{2}}\right] \\
& \leqslant \mathbb{E}_{\Theta_{t}^{\beta}}\left[\frac{\mathbb{1}_{E_{t}^{c}}}{Y_{t}^{\beta}(x)^{2}} \mathbb{I}_{\left\{\varepsilon \sqrt{t} \leqslant Y_{t}^{\beta}(x)\right\}}\right] \\
& +\mathbb{E}_{\Theta_{t}^{\beta}}\left[\frac{1}{Y_{t}^{\beta}(x)^{2}} \mathbb{I}_{\left\{\varepsilon \sqrt{t}>Y_{t}^{\beta}(x\}\right.}\right] \\
& \leqslant \mathbb{E}_{\Theta_{t}^{\beta}}\left[\frac{\mathbb{I}_{E_{t}^{c}}}{Y_{t}^{\beta}(x)^{2}} \mathbb{I}_{\left\{\varepsilon \sqrt{t} \leqslant Y_{t}^{\beta}(x\}\right.}\right] \\
& +\frac{1}{\beta} \mathbb{E}\left[\frac{\mathbb{I}_{\left\{\tau_{x}^{\beta}>t\right\}}}{Y_{t}^{\beta}(x)} \mathbb{I}_{\left\{\varepsilon \sqrt{t}>Y_{t}^{\beta}(x)\right\}} e^{\sqrt{2 d} X_{t}(x)-d t}\right] \\
& \leqslant \frac{\mathbb{E}_{\Theta_{t}^{\beta}}\left[\mathbb{1}_{E_{t}^{c}}\right]}{\varepsilon^{2} t}+\frac{1}{\beta} \mathbb{E}\left[\frac{\mathbb{I}_{\left\{\sup _{[0, t]} X_{u}(x) \leqslant \beta\right\}}}{\left(\beta-X_{t}(x)\right)} \mathbb{I}_{\left\{X_{t}(x)>\beta-\varepsilon \sqrt{t}\right\}}\right]
\end{aligned}
$$

Using the joint law of a Brownian motion together with its maximum, we prove that, for some constant $C$ independent of $t, \varepsilon$, we have:

$$
\frac{1}{\beta} \mathbb{E}\left[\frac{\mathbb{I}_{\left\{\sup _{[0, t]} X_{u}(x) \leqslant \beta\right\}}}{\left(\beta-X_{t}(x)\right)} \mathbb{I}_{\left\{X_{t}(x)>\beta-\varepsilon \sqrt{t}\right\}}\right] \leqslant \varepsilon C / t .
$$

Since $\mathbb{E}_{\Theta_{t}^{\beta}}\left[\mathbb{I}_{E_{t}^{c}}\right] \rightarrow 0$ as $t \rightarrow \infty$, we deduce:

$$
\limsup _{t \rightarrow \infty} t \mathbb{E}_{Q^{\beta}}\left[\xi_{t}^{2}\right] \leqslant \varepsilon C .
$$

Since $\varepsilon$ can be chosen arbitrarily small, the proof of the lemma follows.

Proof of Lemma 14. We consider a function $h$ such that $h_{t} \rightarrow \infty$ as $t \rightarrow \infty$. We define

$$
\begin{array}{llrl}
R_{t}^{\beta, h}(x) & =\int_{B\left(x, e^{-h_{t}}\right) \cap A} \mathbb{1}_{\left\{\tau_{z}^{\beta}>t\right\}} e^{\sqrt{2 d} X_{t}(z)-d t} d z & R_{t}^{\beta, h, c}(x) & =R_{t}^{\beta}(A)-R_{t}^{\beta, h}(x) \\
Z_{t}^{\beta, h}(x) & =\int_{B\left(x, e^{-h_{t}}\right) \cap A} f_{t}^{\beta}(z) d z & Z_{t}^{\beta, h, c}(x) & =Z_{t}^{\beta}(A)-Z_{t}^{\beta, h}(x) .
\end{array}
$$

Now we precise the choice of the set $E_{t}$ :

$$
\begin{aligned}
& E_{t}^{1}=\left\{h_{t}^{1 / 3} \leqslant Y_{h_{t}}^{0}(x) \leqslant h_{t}\right\} \cap\left\{h_{t}^{1 / 6} \leqslant \inf _{u \in[h t, t]} Y_{u}^{0}(x)\right\} \\
& E_{t}^{2}=\left\{Z_{t}^{\beta, h}(x) \leqslant t^{-2}\right\} \\
& E_{t}^{3}=\left\{R_{t}^{\beta, h, c}(x) \leqslant Z_{t}^{\beta, h, c}(x)\right\} \cap\left\{R_{t}^{\beta, h}(x) \leqslant Z_{t}^{\beta, h}(x)\right\} \\
& E_{t}=E_{t}^{1} \cap E_{t}^{2} \cap E_{t}^{3}
\end{aligned}
$$


Let us admit for a while the following lemma:

Lemma 15. Let $\beta>0$. There is a constant $a>0$ such that for every function $h_{t}$ satisfying

$$
\lim _{t \rightarrow \infty} \frac{h_{t}}{\ln t^{a}}=+\infty
$$

and

$$
\lim _{t \rightarrow \infty} \frac{h_{t}}{\sqrt{t}}=0
$$

then

$$
\lim _{t \rightarrow \infty} \Theta_{t}^{\beta}\left(E_{t}\right)=1, \quad \lim _{t \rightarrow \infty} \inf _{u \in\left[h_{t}^{1 / 3}, h_{t}\right]} \Theta_{t}^{\beta}\left(E_{t} \mid Y_{h_{t}}^{0}(x)=u\right)=1 .
$$

So we pursue the proof of Lemma 14 while assuming now that the conditions and conclusions of Lemma 15 are in force. In what follows, $C$ will denote a constant that may change from line to line and that does not depend on relevant quantities. On $E_{t}$, we have $R_{t}^{\beta, h} \leqslant Z_{t}^{\beta, h} \leqslant t^{-2}$. Therefore

$$
\mathbb{E}_{\Theta_{t}^{\beta}}\left[\frac{R_{t}^{\beta, h}}{Z_{t}^{\beta}(A)} \frac{1}{Y_{t}^{\beta}(x)} \mathbb{I}_{E_{t}}\right] \leqslant t^{-2} \mathbb{E}_{\Theta_{t}^{\beta}}\left[\frac{1}{Z_{t}^{\beta}(A)} \frac{1}{Y_{t}^{\beta}(x)} \mathbb{I}_{E_{t}}\right] .
$$

On $E_{t}$, in particular on $E_{t}^{1}$, we have $Y_{t}^{\beta}(x) \geqslant \beta+h_{t}^{1 / 6}$. Hence

$$
\begin{aligned}
\mathbb{E}_{\Theta_{t}^{\beta}}\left[\frac{R_{t}^{\beta, h}}{Z_{t}^{\beta}(A)} \frac{1}{Y_{t}^{\beta}(x)} \mathbb{I}_{E_{t}}\right] & \leqslant t^{-2} \mathbb{E}_{\Theta_{t}^{\beta}}\left[\frac{1}{Z_{t}^{\beta}(A)} \frac{1}{\left(\beta+h_{t}^{1 / 6}\right)}\right] \\
& \leqslant \frac{1}{t^{2}\left(\beta+h_{t}^{1 / 6}\right)} \mathbb{E}_{Q^{\beta}}\left[\frac{1}{Z_{t}^{\beta}(A)}\right] \\
& \leqslant \frac{1}{\beta^{2}|A| t^{2}} .
\end{aligned}
$$

Here we have used $\mathbb{E}_{Q^{\beta}}\left[\frac{1}{Z_{t}^{\beta}(A)}\right]=\frac{1}{\beta|A|}$. Thus we have

$$
\mathbb{E}_{\Theta_{t}^{\beta}}\left[\frac{R_{t}^{\beta, h}}{Z_{t}^{\beta}(A)} \frac{1}{Y_{t}^{\beta}(x)} \mathbb{I}_{E_{t}}\right]=o\left(\frac{1}{t}\right) .
$$

Let us treat the quantity:

$$
\begin{aligned}
\mathbb{E}_{\Theta_{t}^{\beta}} & {\left[\frac{R_{t}^{\beta, h, c}}{Z_{t}^{\beta}(A)} \frac{1}{Y_{t}^{\beta}(x)} \mathbb{I}_{E_{t}}\right] } \\
& =\frac{1}{\beta|A|} \int_{A} \mathbb{E}\left[\frac{R_{t}^{\beta, h, c}(x)}{Z_{t}^{\beta}(A)} \frac{1}{Y_{t}^{\beta}(x)} \mathbb{I}_{E_{t}} f_{t}^{\beta}(x)\right] d x \\
& \leqslant \frac{1}{\beta|A|} \int_{A} \mathbb{E}\left[\frac{R_{t}^{\beta, h, c}(x)}{Z_{t}^{\beta, h, c}(x)} \frac{1}{Y_{t}^{\beta}(x)} \mathbb{I}_{\left\{h_{t}^{1 / 3} \leqslant Y_{h_{t}}^{0}(x) \leqslant h_{t}\right\}} f_{t}^{\beta}(x)\right] d x .
\end{aligned}
$$


We know that the covariance kernel $k$ appearing in (6) vanishes outside a compact set. Without loss of generality, we may assume that $k$ vanishes outside the ball $B(0,1)$. Let us introduce the sigma algebra $\mathcal{G}_{t}$ generated by the random variables $\left\{X_{s}(y) ; y \in \mathbb{R}^{d}, s \leqslant t\right\}$. Conditionally to $x, \mathcal{G}_{h_{t}}$, the random variables $\frac{R_{t}^{\beta, h, c}(x)}{Z_{t}^{\beta, h, c}(x)}$ and $\frac{1}{Y_{t}^{\beta}(x)} f_{t}^{\beta}(x)$ are independent. Therefore we have:

$$
\begin{aligned}
\mathbb{E}_{\Theta_{t}^{\beta}} & \left.\frac{R_{t}^{\beta, h, c}(x)}{Z_{t}^{\beta}(A)} \frac{1}{Y_{t}^{\beta}(x)} \mathbb{I}_{E_{t}}\right] \\
& \leqslant \mathbb{E}_{\Theta_{t}^{\beta}}\left[\frac{R_{t}^{\beta, h, c}(x)}{Z_{t}^{\beta, h, c}(x)} \frac{1}{Y_{t}^{\beta}(x)} \mathbb{I}_{\left\{h_{t}^{1 / 3} \leqslant Y_{h_{t}}^{0}(x) \leqslant h_{t}\right\}}\right] \\
& =\mathbb{E}_{\Theta_{t}^{\beta}}\left[\mathbb{I}_{\left\{h_{t}^{1 / 3} \leqslant Y_{h_{t}}^{0}(x) \leqslant h_{t}\right\}} \mathbb{E}_{\Theta_{t}^{\beta}}\left[\frac{R_{t}^{\beta, h, c}(x)}{Z_{t}^{\beta, h, c}(x)} \mid x, \mathcal{F}_{h_{t}}\right] \mathbb{E}_{\Theta_{t}^{\beta}}\left[\frac{1}{Y_{t}^{\beta}(x)} \mid x, \mathcal{F}_{h_{t}}\right]\right]
\end{aligned}
$$

To complete the proof of Lemma 14, we admit for a while the two following lemma, the proof of which are gathered in the next subsubsection.

Lemma 16. We have

$$
\mathbb{E}_{\Theta_{t}^{\beta}}\left[\frac{1}{Y_{t}^{\beta}(x)} \mid x, \mathcal{G}_{h_{t}}\right] \leqslant \sqrt{\frac{2}{\pi t}}
$$

Lemma 17. We have

$$
\mathbb{E}_{\Theta_{t}^{\beta}}\left[\frac{R_{t}^{\beta, h, c}(x)}{Z_{t}^{\beta, h, c}(x)} \mathbb{I}_{\left\{h_{t}^{1 / 3} \leqslant Y_{h_{t}}^{0}(x) \leqslant h_{t}\right\}}\right] \leqslant \sqrt{\frac{2}{\pi t}}(1+\varepsilon(t)),
$$

for some function $\varepsilon$ such that $\lim _{t \rightarrow \infty} \varepsilon(t)=0$.

We conclude the proof of Lemma 14. With the help Lemma of 16 and 17, we obtain:

$$
\begin{aligned}
\mathbb{E}_{\Theta_{t}^{\beta}} & {\left[\mathbb{I}_{\left\{h_{t}^{1 / 3} \leqslant Y_{h_{t}}^{0}(x) \leqslant h_{t}\right\}} \mathbb{E}_{\Theta_{t}^{\beta}}\left[\frac{R_{t}^{\beta, h, c}(x)}{Z_{t}^{\beta, h, c}(x)} \mid x, \mathcal{G}_{h_{t}}\right] \mathbb{E}_{\Theta_{t}^{\beta}}\left[\frac{1}{Y_{t}^{\beta}(x)} \mid x, \mathcal{G}_{h_{t}}\right]\right] } \\
& \leqslant \sqrt{\frac{2}{\pi t}} \mathbb{E}_{\Theta_{t}^{\beta}}\left[\mathbb{I}_{\left\{h_{t}^{1 / 3} \leqslant Y_{h_{t}}^{0}(x) \leqslant h_{t}\right\}} \mathbb{E}_{\Theta_{t}^{\beta}}\left[\frac{R_{t}^{\beta, h, c}(x)}{Z_{t}^{\beta, h, c}(x)} \mid x, \mathcal{G}_{h_{t}}\right]\right] \\
& =\sqrt{\frac{2}{\pi t}} \mathbb{E}_{\Theta_{t}^{\beta}}\left[\mathbb{I}_{\left\{h_{t}^{1 / 3} \leqslant Y_{h_{t}}^{0}(x) \leqslant h_{t}\right\}} \frac{R_{t}^{\beta, h, c}(x)}{Z_{t}^{\beta, h, c}(x)}\right] \\
& \leqslant \frac{2}{\pi t}(1+\varepsilon(t))
\end{aligned}
$$

for some function $\varepsilon$ such that $\lim _{t \rightarrow \infty} \varepsilon(t)=0$. 


\section{B.2.1 Proofs of auxiliary lemmas}

Proof of Lemma 15. Under $\Theta_{t}^{\beta}$, the process $\left(Y_{s}^{\beta}(x)\right)_{s \leqslant t}$ is a 3-dimensional Bessel process. It is plain to deduce that

$$
\lim _{t \rightarrow \infty} \Theta_{t}^{\beta}\left(E_{t}^{1}\right)=1 .
$$

Let us prove that $\lim _{t \rightarrow \infty} \Theta_{t}^{\beta}\left(E_{t}^{3}\right)=1$. We define the random variables

$$
S_{t}=\sup _{x \in A} X_{t}(x)-\sqrt{2 d} t+\frac{1}{4 \sqrt{2 d}} \ln (t+1), \quad S=\sup _{t \geqslant 0} S_{t},
$$

and the event

$$
B=\{S<+\infty\} .
$$

It is proved in [17] that $\mathbb{P}(B)=1$. It is plain to deduce that $\Theta^{\beta}(B)=1$. We have

$$
\begin{aligned}
Z_{t}^{\beta, h, c}(x) & =\int_{A \backslash B\left(x, e^{-h_{t}}\right)} Y_{t}^{\beta}(z) \mathbb{1}_{\left\{\tau_{z}^{\beta}>t\right\}} e^{\sqrt{2 d} X_{t}(z)-d t} d z \\
& \geqslant \int_{A \backslash B\left(x, e^{-h_{t}}\right)}\left(\beta+\sqrt{2 d} t-\sup _{x \in A} X_{t}(x)\right) \mathbb{1}_{\left\{\tau_{z}^{\beta}>t\right\}} e^{\sqrt{2 d} X_{t}(z)-d t} d z \\
& =\left(\beta+\frac{1}{4 \sqrt{2 d}} \ln (t+1)-S_{t}\right) R_{t}^{\beta, h, c}(x) \\
& \geqslant\left(\beta+\frac{1}{4 \sqrt{2 d}} \ln (t+1)-S\right) R_{t}^{\beta, h, c}(x) .
\end{aligned}
$$

Therefore

$$
\left\{\beta+\frac{1}{4 \sqrt{2 d}} \ln (t+1)-S \geqslant 1\right\} \subset\left\{Z_{t}^{\beta, h, c}(x) \geqslant R_{t}^{\beta, h, c}(x)\right\},
$$

from which we deduce:

$$
\lim _{t \rightarrow \infty} \Theta_{t}^{\beta}\left(Z_{t}^{\beta, h, c}(x) \geqslant R_{t}^{\beta, h, c}(x)\right)=1 .
$$

With the same argument, we prove

$$
\lim _{t \rightarrow \infty} \Theta_{t}^{\beta}\left(Z_{t}^{\beta, h}(x) \geqslant R_{t}^{\beta, h}(x)\right)=1 .
$$

Hence

$$
\lim _{t \rightarrow \infty} \Theta_{t}^{\beta}\left(E_{t}^{3}\right)=1 .
$$

For $E_{t}^{2}$, we will use some computations made in [17]. By using the Markov inequality and by conditioning on an event $B$, we get:

$$
\Theta_{t}^{\beta}\left(Z_{t}^{\beta, h}(x)>t^{-2}\right) \leqslant t^{2} \mathbb{E}_{\Theta_{t}^{\beta}}\left[Z_{t}^{\beta, h}(x) \mid B\right]+\Theta_{t}^{\beta}\left(B^{c}\right)
$$

for every measurable set $B$. We set for $R>0$

$$
B_{R}=\left\{\forall t \geqslant 0 ; \frac{\sqrt{t}}{R(\ln (2+t))^{2}} \leqslant Y_{t}^{\beta}(x) \leqslant R(1+\sqrt{t \ln (1+t)})\right\} .
$$


We choose $B=B_{R}$ in (30). It is proved in [17] that the quantity $\Theta_{t}^{\beta}\left(B_{R}^{c}\right)$ can be made arbitrarily close to 0 when choosing $R$ large enough. Intuitively, this is just a precise statement corresponding to the fact that a Bessel $\beta_{t}$ process goes to infinity with speed rate $\sqrt{t}$ as $t$ goes to $\infty$.

So it just remains to prove that, for $R$ fixed, the quantity $t^{2} \mathbb{E}_{\Theta_{t}^{\beta}}\left[Z_{t}^{\beta, h} \mid B_{R}\right]$ goes to 0 as $t$ goes to $\infty$. To that purpose, it suffices to estimate the quantity

$$
t^{2} \mathbb{E}_{\Theta_{t}^{\beta}}\left[Z_{t}^{\beta, h}(x) \mid\left(X_{s}(x)\right)_{s \leqslant t}, x, B_{R}\right]
$$

and prove that it goes to 0 when $t$ goes to $\infty$ uniformly with respect to the inputs $\left(X_{s}(x)\right)_{s \leqslant t}, x$. We first rewrite this quantity as:

$$
\begin{aligned}
\mathbb{E}_{\Theta_{t}^{\beta}} & {\left[Z_{t}^{\beta, h}(x) \mid\left(X_{s}(x)\right)_{s} \leqslant t, x, B_{R}\right] } \\
\quad= & \mathbb{E}_{\Theta_{t}^{\beta}}\left[\int_{A \cap B\left(x, e^{-h_{t}}\right)} Y_{t}^{\beta}(z) \mathbb{I}_{\left\{\tau_{z}^{\beta}>t\right\}} e^{\sqrt{2 d} X_{t}(z)-d t} d z \mid\left(X_{s}(x)\right)_{s} \leqslant t, x, B_{R}\right] .
\end{aligned}
$$

This expectation has been computed in [17] thanks to explicit formulae for the conditional expectations of the process $\left(X_{s}(z)\right)_{s}$ given $\left(X_{s}(x)\right)_{s \leqslant t}$. Before making these formulae precise, we clarify a few points. The kernel $k$ involved in (6) vanishes outside a compact set. So, without loss of generality, we assume that $k$ vanishes outside the ball $B(0,1)$. We divide the ball $B\left(x, e^{-h_{t}}\right)$ in two areas: the ring $C(t)=B\left(x, e^{-h_{t}}\right) \backslash B\left(x, e^{-t}\right)$ and the ball $B\left(x, e^{-t}\right)$. The results of the computations are the following. Concerning the ring, we have:

$$
\begin{gathered}
\mathbb{E}_{\Theta_{t}^{\beta}}\left[\int_{A \cap C(t)} Y_{t}^{\beta}(z) \mathbb{I}_{\left\{\tau_{z}^{\beta}>t\right\}} e^{\sqrt{2 d} X_{t}(z)-d t} d z \mid\left(X_{s}(x)\right)_{s \leqslant t}, x, B_{R}\right] \\
\quad \leqslant \int_{A \cap C(t)} \frac{e^{D}}{|x-z|^{d}} G\left(\ln \frac{1}{|x-z|}\right) d z
\end{gathered}
$$

for some function $G$ given by

$$
G(y)=(1+\sqrt{y \ln (1+y)})^{2} e^{-\sqrt{2 d} D \frac{\sqrt{y}}{\ln ^{2}(2+y)}}
$$

and some constant $D>0$ depending on irrelevant quantities. Therefore, by making a change of polar coordinates, we get $(C$ stands for a constant, more precisely the area of the $d$-dimensional sphere):

$$
\begin{aligned}
\mathbb{E}_{\Theta_{t}^{\beta}}\left[\int_{A \cap C(t)} Y_{t}^{\beta}(z) \mathbb{I}_{\left\{\tau_{z}^{\beta}>t\right\}} e^{\sqrt{2 d} X_{t}(z)-d t} d z \mid\left(X_{s}(x)\right)_{s} \leqslant t, x, B_{R}\right] \\
\leqslant C \int_{e^{-t}}^{e^{-h_{t}}} \frac{e^{D}}{r^{d}} G\left(\ln \frac{1}{r}\right) r^{d-1} d r \\
\quad=C e^{D} \int_{h_{t}}^{t} G(y) d y .
\end{aligned}
$$


Now we just want to make sure that there exists $a>0$ such that the condition $\lim _{t \rightarrow \infty} \frac{h_{t}}{\ln t^{a}}=$ $+\infty$ implies that the integral $t^{2} e^{D} \int_{h_{t}}^{t} G(y) d y$ goes to 0 as $t \rightarrow \infty$. Observe that for $y$ large enough, the function $G$ is decreasing. Therefore, a rough bound of the quantity under study is

$$
t^{2} e^{D} \int_{h_{t}}^{t} G(y) d y \leqslant t^{3} e^{D} G\left(h_{t}\right) .
$$

Now, for $y$ large enough again, we observe that $G$ is less than $e^{-\sqrt{2 d} D y^{1 / 3}}$. Therefore it is plain to check that any $a>\frac{9}{\sqrt{2 d} D}$ suits.

On the ball $B\left(x, e^{-t}\right)$, we have

$$
\begin{aligned}
\mathbb{E}_{\Theta_{t}^{\beta}}[ & \left.\int_{A \cap B\left(x, e^{-t}\right)} Y_{t}^{\beta}(z) \mathbb{I}_{\left\{\tau_{z}^{\beta}>t\right\}} e^{\sqrt{2 d} X_{t}(z)-d t} d z \mid\left(X_{s}(x)\right)_{s \leqslant t}, x, B_{R}\right] \\
& \leqslant e^{D} G(t)
\end{aligned}
$$

for some irrelevant constant $D$, which may be different from that involved in the bound on the ring. Once again, the condition $\lim _{t \rightarrow \infty} \frac{h_{t}}{\ln t^{a}}=+\infty$ for some well chosen $a>0$ implies that the term $G(t)$ goes to 0 as $t \rightarrow \infty$. Finally we deduce that

$$
\Theta_{t}^{\beta}\left(Z_{t}^{\beta, h}(x) \leqslant t^{-2}\right) \rightarrow 1, \quad \text { as } t \rightarrow \infty .
$$

By gathering $(28)+(29)+(31)$, we have proved that

$$
\Theta_{t}^{\beta}\left(E_{t}\right) \rightarrow 1, \quad \text { as } t \rightarrow \infty \text {. }
$$

It just remains to prove that

$$
\lim _{t \rightarrow \infty} \inf _{u \in\left[h_{t}^{1 / 3}, h_{t}\right]} \Theta_{t}^{\beta}\left(E_{t} \mid Y_{h_{t}}^{0}(x)=u\right)=1 .
$$

We first prove that

$$
\lim _{t \rightarrow \infty} \Theta_{t}^{\beta}\left(\left(E_{t}^{3}\right)^{c} \mid Y_{h_{t}}^{0}(x)=u\right)=0
$$

uniformly with respect to $u \in\left[h_{t}^{1 / 3}, h_{t}\right]$. Observe that

$$
\begin{aligned}
& \Theta_{t}^{\beta}\left(\left(E_{t}^{3}\right)^{c} \mid Y_{h_{t}}^{0}(x)=u\right) \\
& \leqslant \Theta_{t}^{\beta}\left(\left(E_{t}^{3}\right)^{c} \mid Y_{h_{t}}^{0}(x)=u, \sup _{x \in A}-Y_{t}^{0}(x) \leqslant-1\right)+\Theta_{t}^{\beta}\left(\sup _{x \in A}-Y_{t}^{0}(x)>-1\right) \\
& =0+\Theta_{t}^{\beta}\left(\inf _{x \in A} Y_{t}^{0}(x)<1\right) .
\end{aligned}
$$

It results from the observations made at the beginning of the proof of this lemma that the above quantity becomes arbitrarily small as $t$ gets large.

Concerning $E_{t}^{2}$, observe that

$$
\Theta_{t}^{\beta}\left(Z_{t}^{\beta, h}(x)>t^{-2} \mid Y_{h_{t}}^{0}(x)=u\right) \leqslant \Theta_{t}^{\beta}\left(Z_{t}^{\beta, h}(x)>t^{-2} \mid Y_{h_{t}}^{0}(x)=u, B_{R}\right)+\Theta^{\beta}\left(B_{R}^{c}\right) .
$$


Now we observe that we have already proved that $\Theta_{t}^{\beta}\left(Z_{t}^{\beta, h}(x)>t^{-2} \mid Y_{h_{t}}^{0}(x)=u, B_{R}\right) \rightarrow 0$ as $t \rightarrow \infty$. Therefore

$$
\limsup _{t \rightarrow \infty} \sup _{u \in\left[h_{t}^{1 / 3}, h_{t}\right]} \Theta_{t}^{\beta}\left(Z_{t}^{\beta, h}(x)>t^{-2} \mid Y_{h_{t}}^{0}(x)=u\right) \leqslant \Theta^{\beta}\left(B_{R}^{c}\right)
$$

Since $\Theta^{\beta}\left(B_{R}^{c}\right)$ can be made arbitrarily close to 0 for $R$ large enough, we conclude that:

$$
\limsup _{t \rightarrow \infty} \sup _{u \in\left[h_{t}^{1 / 3}, h_{t}\right]} \Theta_{t}^{\beta}\left(Z_{t}^{\beta, h}(x)>t^{-2} \mid Y_{h_{t}}^{0}(x)=u\right)=0 .
$$

It just remains to prove that

$$
\lim _{t \rightarrow \infty} \Theta_{t}^{\beta}\left(E_{t}^{1} \mid Y_{h_{t}}^{0}(x)=u\right)=1
$$

uniformly with respect to $u \in\left[h_{t}^{1 / 3}, h_{t}\right]$. This is obvious since $\left(Y_{t}^{0}\right)_{t}$ is a 3 -dimensional Bessel process.

Proof of Lemma 16. By using standard tricks of changes of probability measures, we have:

$$
\mathbb{E}_{\Theta_{t}^{\beta}}\left[\frac{1}{Y_{t}^{\beta}(x)} \mid x, \mathcal{G}_{h_{t}}\right]=\frac{1}{f_{h_{t}}^{\beta}(x)} \mathbb{E}\left[\frac{f_{t}^{\beta}(x)}{Y_{t}^{\beta}(x)} \mid \mathcal{G}_{h_{t}}\right] .
$$

Let us compute the latter conditional expectation:

$$
\begin{aligned}
\mathbb{E} & {\left[\frac{f_{t}^{\beta}(x)}{Y_{t}^{\beta}(x)} \mid \mathcal{G}_{h_{t}}\right] } \\
& =\mathbb{I}_{\left\{\tau_{x}^{\beta}>h_{t}\right\}} e^{\sqrt{2 d} X_{h_{t}}(x)-d h_{t}} \mathbb{E}\left[\mathbb{I}_{\left\{\sup _{[0, t]} X_{s}(x)-d s \leqslant \beta\right\}} e^{\sqrt{2 d}\left(X_{t}(x)-X_{h_{t}}(x)\right)-d\left(t-h_{t}\right)} \mid \mathcal{G}_{h_{t}}\right] \\
& =\mathbb{I}_{\left\{\tau_{x}^{\beta}>h_{t}\right\}} e^{\sqrt{2 d} X_{h_{t}}(x)-d h_{t}} F\left(Y_{h_{t}}^{\beta}(x)\right)
\end{aligned}
$$

where

$$
F(y)=\mathbb{E}\left[\mathbb{1}_{\left\{\sup _{\left[0, t-h_{t}\right]} X_{s}(x)-d s \leqslant y\right\}} e^{\sqrt{2 d}\left(X_{t-h_{t}}(x)\right)-d\left(t-h_{t}\right)}\right] .
$$

By using the Girsanov transform, we get:

$$
F(y)=\mathbb{E}\left[\mathbb{I}_{\left.\left\{\sup _{\left[0, t-h_{t}\right]} X_{s}(x) \leqslant y\right\}\right]} .\right.
$$

This quantity is plain to compute since the process $s \mapsto X_{s}(x)$ is a Brownian motion:

$$
\begin{aligned}
F(y) & =\mathbb{P}\left(\left|X_{t-h_{t}}(x)\right| \leqslant y\right) \\
& =\frac{1}{\sqrt{2 \pi}} \int_{-\frac{y}{\sqrt{t-h_{t}}}}^{\frac{y}{\sqrt{t-h_{t}}}} e^{-u^{2} / 2} d u \\
& \leqslant \sqrt{\frac{2}{\pi}} \frac{y}{\sqrt{t-h_{t}}} .
\end{aligned}
$$


The lemma follows.

Proof of Lemma 17. We have:

$$
\begin{aligned}
\mathbb{E}_{\Theta_{t}^{\beta}}[ & \left.\frac{R_{t}^{\beta, h, c}(x)}{Z_{t}^{\beta, h, c}(x)} \mathbb{I}_{E_{t}}\right] \\
& \geqslant \mathbb{E}_{\Theta_{t}^{\beta}}\left[\frac{R_{t}^{\beta, h, c}(x)}{Z_{t}^{\beta, h, c}(x)} \mathbb{I}_{\left\{h_{t}^{1 / 3} \leqslant Y_{h_{t}}^{0}(x) \leqslant h_{t}\right\}}\right] \inf _{u \in\left[h_{t}^{1 / 3}, h_{t}\right]} \Theta_{t}^{\beta}\left(E_{t} \mid Y_{h_{t}}^{0}(x)=u\right)
\end{aligned}
$$

From Lemma 15, we have $\Theta_{t}^{\beta}\left(E_{t} \mid Y_{h_{t}}^{0}(x)=u\right) \rightarrow 1$ as $t \rightarrow \infty$ uniformly with respect to $u \in\left[h_{t}^{1 / 3}, h_{t}\right]$. Therefore

$$
\mathbb{E}_{\Theta_{t}^{\beta}}\left[\frac{R_{t}^{\beta, h, c}(x)}{Z_{t}^{\beta, h, c}(x)} \mathbb{I}_{\left\{h_{t}^{1 / 3} \leqslant Y_{h_{t}}^{0}(x) \leqslant h_{t}\right\}}\right] \leqslant(1+\varepsilon(t)) \mathbb{E}_{\Theta_{t}^{\beta}}\left[\frac{R_{t}^{\beta, h, c}(x)}{Z_{t}^{\beta, h, c}(x)} \mathbb{I}_{E_{t}}\right]
$$

for some function $\varepsilon$ such that $\lim _{t \rightarrow \infty} \varepsilon(t)=0$. On $E_{t}$ we have $R_{t}^{\beta, h, c}(x) \leqslant Z_{t}^{\beta, h, c}(x)$, from which we get:

$$
\mathbb{E}_{\Theta_{t}^{\beta}}\left[\frac{R_{t}^{\beta, h, c}(x)}{Z_{t}^{\beta, h, c}(x)} \mathbb{I}_{E_{t}}\right] \leqslant \mathbb{E}_{\Theta_{t}^{\beta}}\left[\frac{R_{t}^{\beta, h, c}(x)}{Z_{t}^{\beta, h, c}(x)} \mathbb{I}_{E_{t} \cap\left\{Z_{t}^{\beta}(A)>1 / t\right\}}\right]+\mathbb{E}_{\Theta_{t}^{\beta}}\left[\mathbb{I}_{\left\{Z_{t}^{\beta}(A) \leqslant 1 / t\right\}}\right] .
$$

By using the Markov inequality, we have:

$$
\mathbb{E}_{Q^{\beta}}\left[\mathbb{1}_{\left\{Z_{t}^{\beta}(A) \leqslant 1 / t\right\}}\right] \leqslant \frac{1}{t} \mathbb{E}_{Q^{\beta}}\left[\frac{1}{Z_{t}^{\beta}(A)}\right] \leqslant \frac{1}{t} .
$$

By gathering (33) and (34), we deduce

$$
\mathbb{E}_{\Theta_{t}^{\beta}}\left[\frac{R_{t}^{\beta, h, c}(x)}{Z_{t}^{\beta, h, c}(x)} \mathbb{I}_{E_{t}}\right] \leqslant \mathbb{E}_{\Theta_{t}^{\beta}}\left[\frac{R_{t}^{\beta, h, c}(x)}{Z_{t}^{\beta, h, c}(x)} \mathbb{I}_{E_{t} \cap\left\{Z_{t}^{\beta}(A)>1 / t\right\}}\right]+\frac{1}{t} .
$$

Since $Z_{t}^{\beta, h}(x) \mathbb{I}_{E_{t}} \leqslant t^{-2}$, we have on the set $E_{t} \cap\left\{Z_{t}^{\beta}(A)>1 / t\right\}$ the estimate $Z_{t}^{\beta, h}(x) \leqslant \frac{1}{t} Z_{t}^{\beta}(A)$. Therefore, on the set $E_{t} \cap\left\{Z_{t}^{\beta}(A)>1 / t\right\}$, we have

$$
Z_{t}^{\beta, h, c}(x) \geqslant\left(1-t^{-1}\right) Z_{t}^{\beta}(A) .
$$

We deduce

$$
\begin{aligned}
\mathbb{E}_{\Theta_{t}^{\beta}}\left[\frac{R_{t}^{\beta, h, c}(x)}{Z_{t}^{\beta, h, c}(x)} \mathbb{I}_{E_{t}}\right] & \leqslant \frac{1}{1-t^{-1}} \mathbb{E}_{\Theta_{t}^{\beta}}\left[\frac{R_{t}^{\beta, h, c}(x)}{Z_{t}^{\beta}(A)} \mathbb{I}_{E_{t} \cap\left\{Z_{t}^{\beta}(A)>1 / t\right\}}\right]+\frac{1}{t} \\
& \leqslant \frac{1}{1-t^{-1}} \mathbb{E}_{\Theta_{t}^{\beta}}\left[\frac{R_{t}^{\beta}(A)}{Z_{t}^{\beta}(A)}\right]+\frac{1}{t} .
\end{aligned}
$$

The result follows by gathering $(17)+(32)+(35)$. 


\section{B.3 Proof of Theorem 5}

In the previous subsection, we have proved the convergence of $\sqrt{t} \frac{R_{t}^{\beta}(A)}{Z_{t}^{\beta}(A)}$ in probability towards $\sqrt{\frac{2}{\pi}}$ under the measure $Q^{\beta}$ for any $\beta>0$. This is the content of Proposition 11 . Our objective is now to use this convergence under $Q^{\beta}$ to establish the convergence under the original probability measure $\mathbb{P}$.

Proposition 11 ensures that, for any $\varepsilon>0$,

$$
\mathbb{Q}^{\beta}\left(\left|\sqrt{t} \frac{R_{t}^{\beta}(A)}{Z_{t}^{\beta}(A)}-\sqrt{\frac{2}{\pi}}\right|>\sqrt{\frac{2}{\pi}} \varepsilon\right) \rightarrow 0, \quad \text { as } t \rightarrow \infty .
$$

Equivalently

$$
\mathbb{E}\left(Z_{t}^{\beta}(A) \mathbb{I}_{\left\{\left|\sqrt{t} \frac{R_{t}^{\beta}(A)}{Z_{t}^{\beta}(A)}-\sqrt{\frac{2}{\pi}}\right|>\sqrt{\frac{2}{\pi}} \varepsilon\right\}}\right) \rightarrow 0, \text { as } t \rightarrow \infty .
$$

From [17], we know that

$$
\sup _{t} \max _{x \in A} X_{t}(x)-\sqrt{2 d} t<\infty
$$

almost surely. By setting

$$
E_{R}=\left\{\sup _{t} \max _{x \in A} X_{t}(x)-\sqrt{2 d} t<R\right\},
$$

we obtain an increasing family such that

$$
\mathbb{P}\left(\bigcup_{R>0} E_{R}\right)=1
$$

From the convergence $\left(Z_{t}^{\beta}(A)\right.$ is nonnegative)

$$
\mathbb{E}\left(Z_{t}^{\beta}(A) \mathbb{I}_{\left\{\left|\sqrt{t} \frac{R_{t}^{\beta}(A)}{Z_{t}^{\beta}(A)}-\sqrt{\frac{2}{\pi}}\right|>\sqrt{\frac{2}{\pi}} \varepsilon\right\}} \mathbb{I}_{E_{R}}\right) \rightarrow 0, \quad \text { as } t \rightarrow \infty,
$$

we deduce the convergence of

$$
Z_{t}^{\beta}(A) \mathbb{I}_{\left\{\left|\sqrt{t} \frac{R_{t}^{\beta}(A)}{Z_{t}^{\beta}(A)}-\sqrt{\frac{2}{\pi}}\right|>\sqrt{\frac{2}{\pi}} \varepsilon\right\}} \mathbb{I}_{E_{R}} \rightarrow 0
$$

in probability as $t \rightarrow \infty$. Fix $\beta>R$. On $E_{R}$ we have $R_{t}^{\beta}(A)=M_{t}^{\sqrt{2 d}}(A)$. Concerning $Z_{t}^{\beta}(A)$, we observe that, for $\beta>R$, we have

$$
\forall t>0, \quad \beta M_{t}^{\sqrt{2 d}}(A)+M_{t}^{\prime}(A)=Z_{t}^{\beta}(A) .
$$

Therefore $\lim _{t \rightarrow} Z_{t}^{\beta}(A)=M^{\prime}(A)>0$ on $E_{R}$ for $\beta>R$ (recall that $M_{t}^{\sqrt{2 d}}(A) \rightarrow 0$ as $t \rightarrow \infty)$. From (36), we deduce that, necessarily on $E_{R}$ :

$$
\mathbb{I}_{\left\{\left|\sqrt{t} \frac{R_{t}^{\beta}(A)}{Z_{t}^{\beta}(A)}-\sqrt{\frac{2}{\pi}}\right|>\sqrt{\frac{2}{\pi}} \varepsilon\right\}} \rightarrow 0, \quad \text { as } t \rightarrow \infty
$$


that is

$$
\mathbb{I}_{\left\{\left|\sqrt{t} \frac{M_{t}^{\sqrt{2 d}}(A)}{M_{t}^{\prime}(A)}-\sqrt{\frac{2}{\pi}}\right|>\sqrt{\frac{1}{\pi}} \varepsilon\right\}} \rightarrow 0, \quad \text { as } t \rightarrow \infty .
$$

The proof of Theorem 5 is over.

\section{B.4 Other proofs of Section 4.}

We use the comparison with multiplicative cascades set out in the appendix of [17]. The idea is to compare moments of discrete lognormal multiplicative cascades to moments of the family $\left(M_{t}\right)_{t}$ thanks to Lemma 10. More precisely and sticking to the notations in [17], we have

$$
\mathbb{E}\left[\left(Z \sqrt{n} M_{n \ln 2}^{\sqrt{2 d}}\left([0,1]^{d}\right)\right)^{\alpha}\right] \leqslant \mathbb{E}\left[\left(\sqrt{n} \int_{T} e^{\bar{X}_{n}(t)-\frac{1}{2} \mathbb{E}\left[\bar{X}_{n}(t)^{2}\right]} \sigma(d t)\right)^{\alpha}\right]
$$

where

- $Z$ is a Gaussian random variable with fixed mean and variance (thus independent of $n$ ), and independent of the family $\left(M_{t}\right)_{t}$,

- the parameter $\alpha$ belongs to ]0,1[, making the mapping $x \mapsto x^{\alpha}$ concave,

- $\int_{T} e^{\bar{X}_{n}(t)-\frac{1}{2} \mathbb{E}\left[\bar{X}_{n}(t)^{2}\right]} \sigma(d t)$ stands for a lognormal multiplicative cascade at generation $n$, the parameters of which are adjusted to be in the critical situation (see [17] for a precise definition).

It is now well established that the right-hand side of (37) is bounded uniformly with respect to $n$. The reader may consult $[1,8,32]$ for instance about this topic. By the Fatou lemma and Theorem 5, we have

$$
\mathbb{E}\left[\left(M^{\prime}\left([0,1]^{d}\right)\right)^{\alpha}\right] \leqslant \liminf _{n \rightarrow \infty} \mathbb{E}\left[\left(\sqrt{n} M_{n \ln 2}^{\sqrt{2 d}}\left([0,1]^{d}\right)\right)^{\alpha}\right]
$$

This shows that the measure $M^{\prime}$ possesses moments of order $q$ for $0 \leqslant q<1$. We already know that it possesses moments of negative order [17].

It remains to prove that

$$
\sup _{t \geqslant 1} \mathbb{E}\left[\left(\frac{1}{\sqrt{t} M_{t}^{\sqrt{2 d}}(A)}\right)^{q}\right]<+\infty .
$$

We write the proof in dimension 1 (generalization to higher dimensions is straightforward) and we assume that the kernel $k$ of (6) vanishes outside the interval $[-1,1]$. Our proof is based on an argument in [46], which we adapt here to get bounds that are uniform in 
t. We work with the ball $A=[0,1]$. The first step consists in writing an appropriate decomposition for the measure $\sqrt{t} M_{t}^{\sqrt{2 d}}([0,1])$. We have:

$$
\begin{aligned}
M_{t+\ln 8}^{\sqrt{2 d}}([0,1]) & =\int_{0}^{1} e^{\sqrt{2} X_{t+\ln 8}(x)-(t+\ln 8)} d x \\
& \geqslant \sum_{k=0}^{3} \int_{\frac{k}{4}}^{\frac{2 k+1}{8}} e^{\sqrt{2} X_{t+\ln 8}(x)-(t+\ln 8)} d x \\
& \geqslant \sum_{k=0}^{3} \inf _{x \in\left[\frac{k}{4}, \frac{2 k+1}{8}\right]} e^{\sqrt{2} X_{\ln 8}(x)-\ln 8} \int_{\frac{k}{4}}^{\frac{2 k+1}{8}} e^{\sqrt{2}\left(X_{t+\ln 8}-X_{\ln 8}\right)(x)-t} d x .
\end{aligned}
$$

We set

$$
Y_{i}=\frac{1}{8} \inf _{x \in\left[\frac{i}{4}, \frac{2 i+1}{8}\right]} e^{\sqrt{2} X_{\ln 8}(x)-\ln 8}, \quad N_{i}=8 \int_{\frac{i}{4}}^{\frac{2 i+1}{8}} e^{\sqrt{2}\left(X_{t+\ln 8}-X_{\ln 8}\right)(x)-t} d x .
$$

for $i=0, \ldots, 3$. A straightforward computation of covariances shows that $\left(\left(X_{t+\ln 8}-\right.\right.$ $\left.\left.X_{\ln 8}\right)(x)\right)_{x \in \mathbb{R}}$ has same distribution as $\left(X_{t}(8 x)\right)_{x \in \mathbb{R}}$. It is plain to deduce that

- the random variables $\left(Y_{i}\right)_{i}$ are independent of $\left(N_{i}\right)_{i}$,

- the random variables $\left(Y_{i}\right)_{i}$ are identically distributed,

- the random variables $\left(N_{i}\right)_{i}$ are identically distributed with common law $M_{t}^{\sqrt{2 d}}([0,1])$.

Let us define

$$
\varphi_{t}(s)=\mathbb{E}\left[e^{-s \sqrt{t} M_{t}^{\sqrt{2 d}}([0,1])}\right]
$$

Since the mapping $x \mapsto e^{-s \sqrt{t} x}$ is convex, we can apply Lemma 10 and obtain

$$
\varphi_{t}(s) \leqslant \mathbb{E}\left[e^{-s \sqrt{t} M_{t+\ln 8}^{\sqrt{2 d}}([0,1])}\right] .
$$

Therefore:

$$
\begin{aligned}
\varphi_{t}(s) & \leqslant \mathbb{E}\left[e^{-s \sqrt{t} M_{t+\ln 8}^{\sqrt{2 d}}([0,1])}\right] \\
& \leqslant \mathbb{E}\left[e^{-s \sqrt{t} \sum_{i=0}^{3} Y_{i} N_{i}}\right] \\
& \left.=\mathbb{E}\left[\prod_{i=0}^{3} \varphi_{t}\left(s Y_{i}\right)\right)\right] \\
& \leqslant \mathbb{E}\left[\varphi_{t}\left(s Y_{1}\right)^{4}\right] .
\end{aligned}
$$

Let us denote by $F$ the distribution function of $Y_{1}$. We have:

$$
\begin{aligned}
\varphi_{t}(s) & \leqslant \int_{0}^{\infty} \varphi_{t}(s x)^{4} F(d x) \\
& =\int_{0}^{s^{-1 / 2}} \varphi_{t}(s x)^{4} F(d x)+\int_{s^{-1 / 2}}^{\infty} \varphi_{t}(s x)^{4} F(d x) \\
& \leqslant F\left(s^{-1 / 2}\right)+\varphi_{t}\left(s^{1 / 2}\right)^{4} .
\end{aligned}
$$


It is plain to check that $\mathbb{E}\left[\left(Y_{1}\right)^{-q}\right]<+\infty$ for all $q>0$. Fix $q_{0}>0$. By the Markov inequality, we deduce:

$$
\varphi_{t}(s) \leqslant s^{-\frac{q_{0}}{2}} \mathbb{E}\left[\left(Y_{1}\right)^{-q_{0}}\right]+\varphi_{t}\left(s^{1 / 2}\right)^{4} .
$$

Therefore

$$
\varphi_{t}(s) \leqslant\left(s^{2 q-\frac{q_{0}}{2}} \mathbb{E}\left[\left(Y_{1}\right)^{-q_{0}}\right]+\varphi_{t}\left(s^{1 / 2}\right)^{2}\right)\left(s^{-2 q}+\varphi_{t}\left(s^{1 / 2}\right)^{2}\right) .
$$

for all $q<q_{0} / 4$. Let us admit for a while the following lemma

Lemma 18. The family of functions $\left(\varphi_{t}(\cdot)\right)_{t}$ uniformly converges on $\mathbb{R}_{+}$as $t \rightarrow \infty$ towards

$$
\varphi(s)=\mathbb{E}\left[e^{-s M^{\prime}([0,1])}\right]
$$

Since $\mathbb{P}\left(M^{\prime}([0,1])=0\right)=0$, we deduce $\lim _{s \rightarrow \infty} \varphi(s)=0$. Therefore there exist $s_{0}>0$ and $t_{0}>0$ such that

$$
\forall s>s_{0}, \forall t>t_{0} \quad\left(s^{2 q-\frac{q_{0}}{2}} \mathbb{E}\left[\left(Y_{1}\right)^{-q_{0}}\right]+\varphi_{t}\left(s^{1 / 2}\right)^{2}\right) \leqslant \frac{1}{\sqrt{2}} .
$$

Plugging this estimate into (38) yields:

$$
\forall s>s_{0}, \forall t>t_{0} \quad \varphi_{t}(s) \leqslant \frac{1}{\sqrt{2}}\left(s^{-2 q}+\varphi_{t}\left(s^{1 / 2}\right)^{2}\right) .
$$

Then, by induction, we check that:

$$
\varphi_{t}\left(s^{2^{n}}\right) \leqslant \frac{1}{\sqrt{2}}\left(a_{n+1} s^{-q 2^{n+1}}+\varphi_{t}^{2^{n+1}}\left(s^{1 / 2}\right)\right)
$$

where $a_{1}=1$ and $a_{n+1}=a_{n}^{2}+1$. Let us choose $Q \geqslant \frac{1+\sqrt{5}}{2}$ so as to have $Q^{2}-Q-1 \geqslant 0$. It is then plain to check by induction that $Q^{2^{n}} \geqslant Q+a_{n}$. Let us choose $s=s_{0}+Q^{1 / q}$. Then for all $x>s$, there exists $n \in \mathbb{N}$ such that:

$$
s^{2^{n}} \leqslant x<s^{2^{n+1}} .
$$

In other words,

$$
2^{n} \leqslant \frac{\ln x}{\ln s}<2^{n+1}
$$

Thus we obtain from (39)

$$
\begin{aligned}
\varphi_{t}(x) & \leqslant \varphi\left(s^{2^{n}}\right) \leqslant \frac{1}{\sqrt{2}}\left(Q^{2^{n+1}} s^{-q 2^{n+1}}+\varphi_{t}^{2^{n+1}}\left(s^{1 / 2}\right)\right) \\
& \leqslant \frac{1}{\sqrt{2}}\left(x^{-\alpha}+x^{-\beta}\right)
\end{aligned}
$$

where $\alpha=q-\frac{\ln Q}{\ln s}>0$ and $\beta=-\frac{\ln \varphi_{t}\left(s^{1 / 2}\right)}{\ln s}>0$. Since $\lim _{t \rightarrow \infty} \varphi_{t}(s)=\mathbb{E}\left[e^{-s M^{\prime}([0,1])}\right]$, we can choose $\beta$ arbitrarily close to $\beta_{0}=-\frac{\ln \varphi\left(s^{1 / 2}\right)}{\ln s}>0$. To sum up, we have proved that, for all $\beta<\beta_{0}$, there exists $x_{0}=s_{0}+Q^{1 / q}$ and $t_{0}>0$ such that

$$
\forall x>x_{0}, \forall t>t_{0} \quad \varphi_{t}(x) \leqslant \frac{1}{\sqrt{2}}\left(x^{-\alpha}+x^{-\beta}\right) .
$$


It is straightforward to deduce

$$
\sup _{t \geqslant 1} \mathbb{E}\left[\left(\frac{1}{\sqrt{t} M_{t}^{\sqrt{2 d}}(A)}\right)^{q}\right]<+\infty
$$

for all $0<q<\min \left(\alpha, \beta_{0}\right)$. Put in other words, we have proved the result "only for small $q "$. But, remembering that

$$
\varphi_{t}(x) \leqslant \mathbb{E}\left[\varphi_{t}\left(x Y_{1}\right)^{4}\right]
$$

and $\mathbb{E}\left[Y_{1}^{-q}\right]<+\infty$ for all $q>0$, we can deduce from (40) the result for arbitrary $q$ by induction.

Proof of Lemma 18. Define the family of functions

$$
f_{t}: y \in\left[0,1\left[\mapsto \varphi_{t}\left(\tan \frac{\pi y}{2}\right) .\right.\right.
$$

Since $\mathbb{P}\left(\sqrt{t} M_{t}^{\sqrt{2}}([0,1])=0\right)=0$, it is plain to deduce that $f_{t}$ can be continuously extended to $[0,1]$ by setting $f_{t}(1)=0$ for all $t$. In the same way we define a continuous function on $[0,1]$ by

$$
f: y \in\left[0,1\left[\mapsto \varphi\left(\tan \frac{\pi y}{2}\right)\right.\right.
$$

and $f(1)=0$ (possible because $\mathbb{P}\left(M^{\prime}([0,1])=0\right)=0$ ). The family $\left(f_{t}\right)_{t}$ pointwise converges as $t \rightarrow \infty$ towards $f$. Furthermore, the functions $f_{t}$ are non increasing for all $t$. It is then standard to deduce the uniform convergence. The lemma follows.

\section{Proof of Section 5}

Proof of Proposition $\%$. Consider a solution $M$ of the $\star$-equation (9) with $\omega_{\varepsilon}$ given by (11) and $\gamma^{2}=2 d$. Consider an exponent $0<q<1$, an open bounded set $A$ and $\lambda<1$. From equation (9) (with $\varepsilon=\lambda$ ) and the Jensen inequality we have:

$$
\begin{aligned}
\mathbb{E}\left[\left(M(\lambda A)^{q}\right]\right. & =\mathbb{E}\left[\left(\int_{\lambda A} e^{\gamma X_{\ln \frac{1}{\lambda}}(r)-\frac{\gamma^{2}}{2} \mathbb{E}\left[X_{\ln \frac{1}{\lambda}}(r)^{2}\right]} \lambda^{d} M^{\lambda}(d r)\right)^{q}\right] \\
& \geqslant \lambda^{d q} \mathbb{E}\left[e^{\gamma q X_{\ln \frac{1}{\lambda}}(0)-\frac{\gamma^{2} q}{2} \mathbb{E}\left[X_{\left.\ln \frac{1}{\lambda}(0)^{2}\right]} M(A)^{q}\right]}\right. \\
& =\lambda^{\xi(q)} \mathbb{E}\left[M(A)^{q}\right] .
\end{aligned}
$$

Conversely, we use Lemma 10 and equation (9):

$$
\begin{aligned}
\mathbb{E}\left[M(\lambda A)^{q}\right] & =\mathbb{E}\left[\left(\int_{\lambda A} e^{\gamma X_{\ln \frac{1}{\lambda}}(r)-\frac{\gamma^{2}}{2} \mathbb{E}\left[X_{\ln \frac{1}{\lambda}}(r)^{2}\right]} M^{\lambda}(d r)\right)^{q}\right] \\
& \leqslant \mathbb{E}\left[\left(\int_{\lambda A} e^{\left.\left.\gamma \sqrt{a_{\lambda} Z-\frac{\gamma^{2}}{2} a_{\lambda}} M^{\lambda}(d r)\right)^{q}\right]}\right.\right.
\end{aligned}
$$


where $Z$ is a standard Gaussian random variable and $a_{\lambda}=\inf _{x, y \in \lambda A} K_{\ln \frac{1}{\lambda}}(y-x)$. Therefore:

$$
\mathbb{E}\left[M(\lambda A)^{q}\right] \leqslant \lambda^{d q} \mathbb{E}\left[e^{\gamma q \sqrt{a_{\lambda}} Z-\frac{q \gamma^{2}}{2} a_{\lambda}}\right] \mathbb{E}\left[M(A)^{q}\right]
$$

We complete the proof by noticing that $a_{\lambda} \geqslant C+\ln \frac{1}{\lambda}$.

Remark 19. Actually, using the $\star$-equation to compute the power-law spectrum is not necessary: it can be computed with similar arguments for any derivative martingale associated to a log-correlated Gaussian field.

Proof of Theorem 8. Without loss of generality, we assume that $k$ vanishes outside the ball $B(0,1)$. Let $K$ be a compact set included in the ball $B(0,1)$ with Lebesgue Hausdorff $\operatorname{dimension}_{\operatorname{dim}} \operatorname{dim}_{L e b}(K)<1$ (the case $\operatorname{dim}_{L e b}(K)=1$ is obvious). Let $q \in[0,1$ [ be such that $\xi(q)>d \operatorname{dim}_{L e b}(K)$ with $\xi(q)<d$. For $\varepsilon>0$, there is a covering of $K$ by a countable family of balls $\left(B\left(x_{n}, r_{n}\right)\right)_{n}$ such that

$$
\sum_{n} r_{n}^{\xi(q)}<\varepsilon
$$

By using in turn the stationarity and the power law spectrum of the measure, we have

$$
\begin{aligned}
\mathbb{E}\left[\sum_{n} M^{\prime}\left(B\left(x_{n}, r_{n}\right)\right)^{q}\right] & =\sum_{n} \mathbb{E}\left[M^{\prime}\left(B\left(0, r_{n}\right)\right)^{q}\right] \\
& \leqslant C_{q} \sum_{n} r_{n}^{\xi(q)} \\
& \leqslant C_{q} \varepsilon .
\end{aligned}
$$

Using the Markov inequality, we deduce

$$
\mathbb{P}\left(\sum_{n} M^{\prime}\left(B\left(x_{n}, r_{n}\right)\right)^{q} \leqslant C_{q} \sqrt{\varepsilon}\right) \geqslant 1-\sqrt{\varepsilon}
$$

Thus, with probability $1-\sqrt{\varepsilon}$, there is a covering of balls of $K$ such that

$$
\sum_{n} M^{\prime}\left(B\left(x_{n}, r_{n}\right)\right)^{q} \leqslant C_{q} \sqrt{\varepsilon}
$$

So $q \geqslant \operatorname{dim}_{M^{\prime}}(K)$ almost surely. Therefore $d \operatorname{dim}_{L e b}(K) \geqslant \operatorname{dim}_{M}(K)$.

Conversely, consider $q \in\left[0,1\left[\operatorname{such}\right.\right.$ that $\xi(q)<d \operatorname{dim}_{L e b}(K)$. By the Frostman Lemma, there is a probability measure $\gamma$ supported by $K$ such that

$$
\int_{B(0,1)^{2}} \frac{1}{|x-y|^{\xi(q)}} \gamma(d x) \gamma(d y)<+\infty .
$$


For $q \in[0,1[$, let us define the random measure $\widetilde{\gamma}$ as the almost sure limit of the following family of positive random measures:

$$
\widetilde{\gamma}(d x)=\lim _{t \rightarrow \infty} e^{q \sqrt{2 d} X_{t}(x)-q^{2} d \mathbb{E}\left[\left(X_{t}(x)\right)^{2}\right]} \gamma(d x) .
$$

The limit is non trivial: Kahane [34] (see also a more general proof in [51]) proved that, for a Radon measure $\gamma(d x)$ satisfying (41) with a power exponent $\kappa$ (instead of $\xi(q)$ in (41)) the associated chaos

$$
\widetilde{\gamma}(d x)=\lim _{t \rightarrow \infty} e^{s X_{t}(x)-\frac{s^{2}}{2} \mathbb{E}\left[\left(X_{t}(x)\right)^{2}\right]} \gamma(d x)
$$

is non degenerate (i.e. the martingale is regular) provided that $\kappa-\frac{s^{2}}{2}>0$. In our context, this condition reads $q^{2} d<\xi(q)$, that is $q<1$.

From the Frostman lemma again, we just have to prove that the quantity

$$
\int_{B(0,1)^{2}} \frac{1}{M^{\prime}(B(x,|y-x|))^{q}} \widetilde{\gamma}(d x) \widetilde{\gamma}(d y)
$$

is finite almost surely, which will follow from

$$
\mathbb{E}\left[\int_{B(0,1)^{2}} \frac{1}{M^{\prime}(B(x,|y-x|))^{q}} \widetilde{\gamma}(d x) \widetilde{\gamma}(d y)\right]<+\infty .
$$

Actually, by using the Fatou lemma in (43), it is even sufficient to prove that:

$$
\liminf _{t \rightarrow \infty} \int_{B(0,1)^{2}} \mathbb{E}\left[\frac{e^{q \sqrt{2 d} X_{t}(x)_{x}+q \sqrt{2 d} X_{t}(y)-2 q^{2} d \mathbb{E}\left[\left(X_{t}(x)\right)^{2}\right]}}{\left(\sqrt{t} M_{t}^{\sqrt{2 d}}(B(x,|y-x|))\right)^{q}}\right] \gamma(d x) \gamma(d y)<+\infty .
$$

From now on, we will focus on computing the above integral (44). There is a way of making the computations with minimal effort: we change the process $X_{t}$ with the perfect scaling process introduced in [52]. We just have to justify that this change of processes is mathematically rigorous. So let us admit for a while the following lemma:

Lemma 20. If (44) is finite for the process $\left(X_{t}(x)\right)_{t, \geqslant 0, x \in \mathbb{R}^{d}}$ with correlations given by

$$
\mathbb{E}\left[X_{t}(x), X_{s}(y)\right]=\int_{S} g_{e^{-\min (s, t)}}(|\langle x-y, s\rangle|) \sigma(d s)
$$

where $S$ stands for the sphere of $\mathbb{R}^{d}, \sigma$ the uniform measure on the sphere and the function $g_{u}($ for $0<u \leqslant 1)$ is given by

$$
g_{u}(r)= \begin{cases}\ln _{+} \frac{2}{r} & \text { if } r \geqslant u \\ \ln \frac{2}{u}+1-\frac{r}{u} & \text { if } r<u\end{cases}
$$

then (44) is finite for the process $\left(X_{t}(x)\right)_{t, \geqslant 0, x \in \mathbb{R}^{d}}$ with correlations given by (6). 
So, from now on, we assume that the correlations of $\left(X_{t}(x)\right)_{t, \geqslant 0, x \in \mathbb{R}^{d}}$ are the new correlations specified in Lemma 20 (see [52] for further details). Notice also that the measure $M^{\gamma}$ also involves this new process. Such a family of kernels possesses useful scaling properties, namely that for $|x| \leqslant 1$ and $0<\lambda<1, K_{t+h}\left(e^{-h} x\right)=K_{t}(x)+h$. In particular, we have the following scaling relation for all $0<l<1$ and all $0<\lambda<1$ :

$$
\begin{aligned}
\left(\left(X_{t+h}\left(e^{-h} x\right)\right)_{x \in B(0,1)},\right. & \left.\left(M_{t+h}^{\sqrt{2 d}}\left(e^{-h} A\right)\right)_{A \subset B(0,1 T)}\right) \\
& \stackrel{\operatorname{law}}{=}\left(\left(X_{t}(x)+\Omega_{h}\right)_{x \in B(0,1)},\left(e^{\sqrt{2 d} \Omega_{h}-2 d h} M_{t}^{\sqrt{2 d}}(A)\right)_{A \subset B(0,1)}\right) .
\end{aligned}
$$

where $\Omega_{h}$ is a centered Gaussian random variable with variance $h$ and independent of the couple

$$
\left(\left(X_{x}^{t}\right)_{x \in B(0,1)},\left(M_{t}^{\sqrt{2 d}}(A)\right)_{A \subset B(0,1)}\right) .
$$

We will use the above relation throughout the proof.

By stationarity of the process $X_{t}$, we can translate the integrand in the quantity (44) to get

$$
\begin{array}{rl}
\int_{B(0,1)^{2}} & \mathbb{E}\left[\frac{e^{q \sqrt{2 d} X_{t}(x)+q \sqrt{2 d} X_{t}(y)-2 q^{2} d \mathbb{E}\left[\left(X_{t}(x)\right)^{2}\right]}}{\left(\sqrt{t} M_{t}^{\sqrt{2 d}}(B(x,|y-x|))\right)^{q}}\right] \gamma(d x) \gamma(d y) \\
& \leqslant \int_{B(0,1)^{2}} \mathbb{E}\left[\frac{e^{q \sqrt{2 d} X_{t}(0)+q \sqrt{2 d} X_{t}(y-x)-q^{2} d \mathbb{E}\left[\left(X_{t}(0)\right)^{2}\right]}}{\left(\sqrt{t} M_{t}^{\sqrt{2 d}}(B(0,|y-x|))\right)^{q}}\right] \gamma(d x) \gamma(d y) .
\end{array}
$$

Now we split the latter integral according to scales larger or smaller than $e^{-t}$ and obtain

$$
\begin{array}{rl}
\int_{B(0,1)^{2}} & \mathbb{E}\left[\frac{e^{q \sqrt{2 d} X_{t}(0)+q \sqrt{2 d} X_{t}(y-x)-q^{2} d \mathbb{E}\left[\left(X_{t}(0)\right)^{2}\right]}}{\left(\sqrt{t} M_{t}^{\sqrt{2 d}}(B(0,|y-x|))\right)^{q}}\right] \gamma(d x) \gamma(d y) \\
\leqslant & \int_{|x-y|>e^{-t}} \mathbb{E}\left[\frac{e^{q \sqrt{2 d} X_{t}(0)+q \sqrt{2 d} X_{t}(y-x)-q^{2} d \mathbb{E}\left[\left(X_{t}(0)\right)^{2}\right]}}{\left(\sqrt{t} M_{t}^{\sqrt{2 d}}(B(0,|y-x|))\right)^{q}}\right] \gamma(d x) \gamma(d y) \\
& +\int_{|x-y| \leqslant e^{-t}} \mathbb{E}\left[\frac{e^{q \sqrt{2 d} X_{t}(0)+q \sqrt{2 d} X_{t}(y-x)-q^{2} d \mathbb{E}\left[\left(X_{t}(0)\right)^{2}\right]}}{\left(\sqrt{t} M_{t}^{\sqrt{2 d}}(B(0,|y-x|))\right)^{q}}\right] \gamma(d x) \gamma(d y) \\
& \stackrel{\text { def }}{=} I_{1}(t)+I_{2}(t)
\end{array}
$$


We first estimate $I_{1}(t)$. For $|x-y|>e^{-t}$, by using (46) with $e^{-h}=|y-x|$, we deduce

$$
\begin{aligned}
\mathbb{E} & {\left[\frac{e^{q \sqrt{2 d} X_{t}(0)+q \sqrt{2 d} X_{t}(y-x)-q^{2} d \mathbb{E}\left[\left(X_{t}(0)\right)^{2}\right]}}{\left(\sqrt{t} M_{t}^{\sqrt{2 d}}(B(0,|y-x|))\right)^{q}}\right] } \\
& =\mathbb{E}\left[\frac{e^{q 2 \sqrt{2 d} \Omega_{h}-2 q^{2} d h} e^{q \sqrt{2 d} X_{t-h}(0)+q \sqrt{2 d} X_{t-h}\left(\frac{y-x}{|y-x|}\right)-2 q^{2} d \mathbb{E}\left[\left(X_{t-h}(0)\right)^{2}\right]}}{\left(e^{\sqrt{2 d} \Omega_{h}-2 d h} \sqrt{t} M_{t-h}^{\sqrt{2 d}}(B(0,1))\right)^{q}}\right] \\
& =\mathbb{E}\left[e^{q \sqrt{2 d} \Omega_{h}-2\left(q^{2}-q\right) d h}\right] \mathbb{E}\left[\frac{e^{q \sqrt{2 d} X_{t-h}(0)+q \sqrt{2 d} X_{t-h}\left(\frac{y-x}{\mid y-x}\right)-2 q^{2} d \mathbb{E}\left[\left(X_{t-h}(0)\right)^{2}\right]}}{\left(\sqrt{t} M_{t-h}^{\sqrt{2 d}}(B(0,1))\right)^{q}}\right] \\
& \leqslant \frac{C}{|y-x| \xi(q)} \mathbb{E}\left[\frac{1}{\left(\sqrt{t} M_{t-h}^{\sqrt{2 d}}(B(0,1 / 2))\right)^{q}}\right]
\end{aligned}
$$

In the last line, we have used a Girsanov transform to get rid of the numerator. Therefore, if we set $c=\sup _{u \leqslant 1} \mathbb{E}\left[\frac{1}{\left(M_{u}^{\sqrt{2 d}}(B(0,1 / 2))\right)^{q}}\right]$, then

$$
I_{1}(t) \leqslant C\left(\sup _{u \geqslant 1} \mathbb{E}\left[\frac{1}{\left(\sqrt{u} M_{u}^{\sqrt{2 d}}(B(0,1 / 2))\right)^{q}}\right]+\frac{c}{\sqrt{t}}\right) \int_{|x-y|>e^{-t}} \frac{C}{|y-x| \xi(q)} \gamma(d x) \gamma(d y) .
$$

From Corollary 6, the quantity

$$
\sup _{u \geqslant 1} \mathbb{E}\left[\frac{1}{\left(\sqrt{u} M_{u}^{\sqrt{2 d}}(B(0,1 / 2))\right)^{q}}\right]
$$

is finite.

Remark 21. Corollary 6 only deals with Gaussian fields with correlations given by (6). From Kahane's convexity inequality, this quantity is also finite for every Gaussian fields with correlations given

$$
K(x, y)=2 d \ln _{+} \frac{1}{|y-x|}+g(x, y)
$$

for some bounded function $\mathrm{g}$. In particular, it is finite for the field considered in Lemma 20.

To treat the term $I_{2}(t)$, we use quite a similar argument excepted that we use the 
scaling relation on $h=t$ instead of $-\ln |y-x|$, and the Girsanov transform again:

$$
\begin{aligned}
& I_{2}(t) \\
& =\int_{|y-x| \leqslant e^{-t}} \mathbb{E}\left[\frac{e^{2 q \sqrt{2 d} \Omega_{t}-q^{2} 2 d t} e^{q \sqrt{2 d} X_{0}(0)+q \sqrt{2 d} X_{0}\left(e^{t}(y-x)\right)-q^{2} 2 d \mathbb{E}\left[\left(X_{0}(0)\right)^{2}\right]}}{\left(e^{q \sqrt{2 d} \Omega_{t}-q 2 d t} \sqrt{t} M_{0}\left(B\left(0, e^{t}(y-x)\right)\right)\right)^{q}}\right] \gamma(d x) \gamma(d y) \\
& =\int_{|y-x| \leqslant e^{-t}} \mathbb{E}\left[e^{q \sqrt{2 d} \Omega_{t}-2\left(q^{2}-q\right) d t}\right] \mathbb{E}\left[\frac{e^{q \sqrt{2 d} X_{0}(0)+q \sqrt{2 d} X_{0}\left(e^{t}(y-x)\right)-q^{2} 2 d \mathbb{E}\left[\left(X_{0}(0)\right)^{2}\right]}}{\left(\sqrt{t} M_{0}\left(B\left(0, e^{t}(y-x)\right)\right)\right)^{q}}\right] \gamma(d x) \gamma(d y) \\
& =\int_{|y-x| \leqslant e^{-t}} \frac{e^{t \xi(q)}}{t^{q / 2}} \mathbb{E}\left[\frac{e^{q^{2} 2 d K_{0}\left(e^{t}(y-x)\right)}}{\left(\int_{B\left(0, e^{t}(y-x)\right)} e^{\sqrt{2 d} X_{0}(u)-d \mathbb{E}\left[\left(X_{1}(0)\right)^{2}\right]+q 2 d K_{0}\left(e^{t}(y-x)-u\right)+q 2 d K_{0}(u)} d u\right)^{q}}\right] \gamma(d x) \gamma(d y) .
\end{aligned}
$$

By using the fact that $K_{0}$ is positive and bounded by $\ln 2$, we have (for some positive constant $C$ independent of $t$ )

$$
I_{2}(t) \leqslant C \int_{|y-x| \leqslant e^{-t}} \frac{e^{t \xi(q)}}{t^{q / 2}} \mathbb{E}\left[\frac{1}{\left(\int_{B\left(0, e^{t}(y-x)\right)} e^{\sqrt{2 d} X_{0}(u)-d \mathbb{E}\left[\left(X_{1}(0)\right)^{2}\right]} d u\right)^{q}}\right] \gamma(d x) \gamma(d y) .
$$

Since $\mathbb{E}\left[X_{0}(u) X_{0}(0)\right] \leqslant \mathbb{E}\left[\left(X_{0}(0)\right)^{2}\right]$, we can use Kahane's convexity inequalities to the convex mapping $x \mapsto \frac{1}{x^{q}}$. We deduce (for some positive constant $C$ independent of $t$, which may change from line to line)

$$
\begin{aligned}
I_{2}(t) & \leqslant C \int_{|y-x| \leqslant e^{-t}} e^{t \xi(q)} t^{-q / 2} \mathbb{E}\left[\frac{1}{\left(\int_{B\left(0, e^{t}(y-x)\right)} e^{\sqrt{2 d} X_{0}(0)-d \mathbb{E}\left[\left(X_{1}(0)\right)^{2}\right]} d u\right)^{q}}\right] \gamma(d x) \gamma(d y) \\
& \leqslant C \int_{|y-x| \leqslant e^{-t}} e^{t \xi(q)} \frac{1}{e^{d q t}|y-x|^{d q}} \gamma(d x) \gamma(d y) \\
& \leqslant C t^{-q / 2} \int_{|y-x| \leqslant e^{-t}} \frac{1}{(y-x)^{\xi(q)}} \gamma(d x) \gamma(d y) .
\end{aligned}
$$

Hence

$$
\lim _{t} I_{2}(t) \leqslant C \limsup _{t \rightarrow \infty} t^{-q / 2} \int_{|y-x| \leqslant e^{-t}} \frac{1}{(y-x)^{\xi(q)}} \gamma(d x) \gamma(d y)=0
$$

The KPZ formula is proved.

Proof of Lemma 20. Let us denote by $K_{t}$ the $\star$-scale invariant kernel given by (6) associated to the process $\left(X_{t}(x)\right)_{t, x}$. We will use the superscript ${ }^{p}$ to denote the corresponding quantities associated to the "perfect" kernel of [52]: we denote by $K_{t}^{p}$ the kernel described in Lemma 20, by $\left(X_{t}^{p}(x)\right)_{t, x}\left(\operatorname{resp} . M_{t}^{p, \sqrt{2 d}}\right)$ the associated Gaussian field (resp. approximate multiplicative chaos). It is plain to see there is a constant $C>0$ such that

$$
\forall t>0, \forall x \in \mathbb{R}^{d}, \quad K_{t}(x)-C \leqslant K_{t}^{p}(x) \leqslant K_{t}(x)+C .
$$


Now we prove the Lemma. By using the Girsanov transform, we have:

$$
\begin{aligned}
& \int_{B(0,1)^{2}} \mathbb{E}\left[\frac{e^{q \sqrt{2 d} X_{t}(x)+q \sqrt{2 d} X_{t}(y)-2 q^{2} d \mathbb{E}\left[\left(X_{t}(x)\right)^{2}\right]}}{\left(\sqrt{t} M_{t}^{\sqrt{2 d}}(B(x,|y-x|))\right)^{q}}\right] \gamma(d x) \gamma(d y) \\
& =\int_{B(0,1)^{2}} \mathbb{E}\left[\frac{1}{\left(\sqrt{t} \int_{B(x,|y-x|)} e^{q 2 d K_{t}(u-x)+q 2 d K_{t}(u-y)} M_{t}^{\sqrt{2 d}}(d u)\right)^{q}}\right] e^{q^{2} 2 d K_{t}(x-y)} \gamma(d x) \gamma(d y) \\
& \leqslant e^{\left(2 d q^{2}+4 d q\right) C} \int_{B(0,1)^{2}} \mathbb{E}\left[\frac{1}{\left(\sqrt{t} \int_{B(x,|y-x|)} e^{q 2 d K_{t}^{p}(u-x)+q 2 d K_{t}^{p}(u-y)} M_{t}^{\sqrt{2 d}}(d u)\right)^{q}}\right] e^{q^{2} 2 d K_{t}^{p}(x-y)} \gamma(d x) \gamma(d y) .
\end{aligned}
$$

By the Girsanov transform again, we have

$$
\begin{gathered}
\int_{B(0,1)^{2}} \mathbb{E}\left[\frac{1}{\left(\sqrt{t} \int_{B(x,|y-x|)} e^{q 2 d K_{t}^{p}(u-x)+q 2 d K_{t}^{p}(u-y)} M_{t}^{\sqrt{2 d}}(d u)\right)^{q}}\right] e^{q^{2} 2 d K_{t}^{p}(x-y)} \gamma(d x) \gamma(d y) \\
\quad=\int_{B(0,1)^{2}} \mathbb{E}\left[\frac{e^{q \sqrt{2 d} X_{t}^{p}(x)+q \sqrt{2 d} X_{t}^{p}(y)-2 q^{2} d \mathbb{E}\left[\left(X_{t}^{p}(x)\right)^{2}\right]}}{\left(\sqrt{t} M_{t}^{p, \sqrt{2 d}}(B(x,|y-x|))\right)^{q}}\right] \gamma(d x) \gamma(d y) .
\end{gathered}
$$

The lemma follows.

Remark 22. To sum up, we have proved that establishing the KPZ formula for the perfect kernel is equivalent to establishing the KPZ formula for all $\star$-scale invariant kernels. Furthermore the above argument is obviously valid for any log-correlated Gaussian field (as it only involves the Girsanov transform) and for other values of $\gamma$ : for $\gamma^{2}<2 d$ with the techniques developed in [53] or for $\gamma^{2}>2 d$ with the techniques developed in [6]. In particular, the KPZ formula established in [6, 53] in terms of Hausdorff dimensions are valid for the GFF. The reader may compare with [20] where the KPZ formula is stated in terms of expected box counting dimensions. At criticality, things are a bit more subtle: the KPZ formula established in this paper is valid for all the derivative martingale for which you can establish the renormalization theorem 5. This theorem is necessary to be in position to apply Kahane's convexity inequalities.

\section{References}

[1] Aïdékon E., Shi Z.: The Seneta-Heyde scaling for the branching random walk, arXiv:1102.0217v2.

[2] Allez R., Rhodes R., Vargas V.: Lognormal «-scale invariant random measures, Probability Theory and Related Fields, to appear, arXiv:1102.1895v1.

[3] Alvarez-Gaumé L., Barbón, J. L. F., and Crnković Č.: A proposal for strings at D > 1, Nucl. Phys. B394, 383 (1993). 
[4] Ambjørn J., Durhuus B., and Jonsson T.: A solvable 2d gravity model with $\gamma>0$, Mod. Phys. Lett. A 9, 1221 (1994).

[5] Bacry E., Muzy J.F.: Log-infinitely divisible multifractal processes, Comm. Math. Phys., 236 (2003) no.3, 449-475.

[6] Barral J., Jin X., Rhodes R., Vargas V.: Gaussian multiplicative chaos and KPZ duality, arXiv:1202.5296v2.

[7] Barral, J., Mandelbrot, B.B.: Multifractal products of cylindrical pulses, Probab. Theory Relat. Fields 124 (2002), 409-430.

[8] Barral J., Kupiainen A., Nikula M., Saksman E., Webb C.: Critical Mandelbrot's cascades, arXiv:1206.5444v1.

[9] Biggins, J.D. and Kyprianou, A.E.: Fixed points of the smoothing transform; the boundary case, Electronic Journal of Probability, vol. 10 (2005).

[10] Benjamini, I., Schramm, O.: KPZ in one dimensional random geometry of multiplicative cascades, Communications in Mathematical Physics, vol. 289, no 2, 653-662, 2009 .

[11] Bramson M., Ding J., Zeitouni O.: Convergence in law of the maximum of the twodimensional discrete Gaussian free field, arXiv:1301.6669.

[12] Brézin E., Kazakov V.A. , Zamolodchikov Al.B.: Scaling violation in a field theory of closed strings in one physical dimension, Nuclear Physics B338, 673-688 (1990).

[13] Das S. R., Dhar A., Sengupta A. M., and Wadia S. R.: New critical behavior in $d=0$ large- $N$ matrix models, Mod. Phys. Lett. A 5, 1041 (1990).

[14] David, F.: Conformal Field Theories Coupled to 2-D Gravity in the Conformal Gauge, Mod. Phys. Lett. A, 3 (1988).

[15] Di Francesco P., Ginsparg P., Zinn-Justin J.: 2D gravity and random matrices, Physics Reports 254, p. 1-133 (1995).

[16] Distler J., Kawai H.: Conformal Field Theory and 2-D Quantum Gravity or Who's Afraid of Joseph Liouville?, Nucl. Phys. B321 509-517 (1989).

[17] Duplantier B., Rhodes R., Sheffield S., Vargas V.: Critical Gaussian Multiplicative Chaos: convergence of the derivative martingale, arXiv:1206.1671.

[18] Duplantier B.: A rigorous perspective on Liouville quantum gravity and KPZ, in Exact Methods in Low-dimensional Statistical Physics and Quantum Computing, J. Jacobsen, S. Ouvry, V. Pasquier, D. Serban, and L.F. Cugliandolo, eds., Lecture Notes of the Les Houches Summer School: Volume 89, July 2008, Oxford University Press (Clarendon, Oxford) (2010). 
[19] Duplantier B.: Conformal fractal geometry and boundary quantum gravity, in Fractal geometry and applications: a jubilee of Benoît Mandelbrot, Part 2 (Amer. Math. Soc., Providence, RI, 2004), vol. 72 of Proc. Sympos. Pure Math., pp. 365-482, arXiv:mathph/0303034.

[20] Duplantier, B., Sheffield, S.: Liouville Quantum Gravity and KPZ, Inventiones Mathematicae, 2011, 185 (2), 333-393.

[21] Duplantier, B., Sheffield, S.: Duality and KPZ in Liouville Quantum Gravity, Physical Review Letters, 102, 150603 (2009).

[22] Duplantier, B., Sheffield, S.: Schramm-Loewner Evolution and Liouville Quantum Gravity, Physical Review Letters, 107, 131305 (2011).

[23] Durhuus B.: Multi-spin systems on a randomly triangulated surface, Nucl. Phys. B426, 203 (1994).

[24] Durrett R., Liggett T.M.: Fixed points of the smoothing transformation, Probability Theory and Related Fields, 1983, vol 64, n. 3, 275-301.

[25] Falconer K.J.: The geometry of fractal sets, Cambridge University Press, 1985.

[26] Fan A.H., Sur le chaos de Lévy d'indice $0<\alpha<1$, Ann. Sciences Math. Québec, vol 21 no. 1, 1997, p. 53-66.

[27] Ginsparg P. and Moore G.: Lectures on 2D gravity and 2D string theory, in Recent direction in particle theory, Proceedings of the 1992 TASI, edited by J. Harvey and J. Polchinski (World Scientific, Singapore, 1993).

[28] Ginsparg P., Zinn-Justin J.: 2D gravity + 1D matter, Physics Letters B240, 333-340 (1990).

[29] Gross, D.J. and Klebanov I.R.: One-dimensional string theory on a circle, Nuclear Physics B344 (1990) 475-498.

[30] Gross D.J., Miljković N.: A nonperturbative solution of $D=1$ string theory, Physics Letters B238, 217-223 (1990).

[31] Gubser S.S., Klebanov I.R.: A modified $c=1$ matrix model with new critical behavior, Physics Letters B340 (1994) 35-42.

[32] Hu Y., Shi Z.: Minimal position and critical martingale convergence in branching random walks, and directed polymers on disordered trees, Annals of Probability, 37 (2) (2009) 742-789.

[33] Jain S. and Mathur S. D.: World-sheet geometry and baby universes in 2-D quantum gravity, Phys. Lett. B 286, 239 (1992). 
[34] Kahane J.-P.: Sur le chaos multiplicatif, Ann. Sci. Math. Québec, 9 no.2 (1985), 105-150.

[35] Kazakov V., Kostov I., and Kutasov D.: A Matrix Model for the 2d Black Hole, in Nonperturbative Quantum Effects 2000, JHEP Proceedings; A matrix model for the two-dimensional black hole, Nucl. Phys. B622 (2002) 141-188.

[36] Klebanov I.R.: Touching random surfaces and Liouville gravity, Phys. Rev. D 51, 18361841 (1995).

[37] Klebanov I.R., Hashimoto A.: Non-perturbative Solution of Matrix Models Modified by Trace-squared Terms, Nucl. Phys. B434 (1995) 264-282.

[38] Klebanov I.R., Hashimoto A.: Wormholes, Matrix Models, and Liouville Gravity, Nucl. Phys. (Proc. Suppl.) 45B,C (1996) 135-148.

[39] Knizhnik V.G., Polyakov A.M., Zamolodchikov A.B.: Fractal structure of 2Dquantum gravity, Modern Phys. Lett A, 3(8) (1988), 819-826.

[40] Kostov I.K.: Loop amplitudes for nonrational string theories, Phys. Lett. B266, 317-324 (1991).

[41] Kostov I.K.: Strings with discrete target space, Nucl. Phys. B376, 539-598 (1992).

[42] Kostov I.K.: Boundary Loop Models and and 2D Quantum Gravity, in Exact Methods in Low-dimensional Statistical Physics and Quantum Computing, J. Jacobsen, S. Ouvry, V. Pasquier, D. Serban, and L.F. Cugliandolo, eds., Lecture Notes of the Les Houches Summer School: Volume 89, July 2008, Oxford University Press (Clarendon, Oxford) (2010).

[43] Kostov I.K., Staudacher M.: Multicritical phases of the $O(n)$ model on a random lattice, Nucl. Phys. B384, 459-483 (1992).

[44] Lalley S.P., Sellke T.: A conditional limit theorem for frontier of a branching Brownian motion, Ann. Probab. 15, 1987, 1052-1061.

[45] Q.S. Liu, Fixed points of a generalized smoothing transformation and applications to the branching random walk, Adv. Appl. Probab. 30 (1998), 85-112.

[46] Molchan, G.M.: Scaling exponents and multifractal dimensions for independent random cascades, Communications in Mathematical Physics, 179 (1996), 681-702.

[47] Motoo M.: Proof of the iterated logarithm through diffusion equation, Ann. Inst. Statist. Math., 10 (1959) 21-28.

[48] Nakayama Y.: Liouville Field Theory - A decade after the revolution, Int. J. Mod. Phys. A19, 2771 (2004). 
[49] Parisi G.: On the one dimensional discretized string, Physics Letters B238, 209-212 (1990).

[50] Polchinski J., Critical behavior of random surfaces in one dimension, Nuclear Physics B346 (1990) 253-263.

[51] Rhodes R., Sohier J., and Vargas V.: «-scale invariant random measures, to appear in Annals of Probability, arXiv:1201.5219v1.

[52] Rhodes R., Vargas, V.: Multidimensional multifractal random measures, Electronic Journal of Probability, 15 (2010), 241-258.

[53] Rhodes, R. Vargas, V.: KPZ formula for log-infinitely divisible multifractal random measures, ESAIM Probability and Statistics, 15, 2011, 358-371.

[54] Robert, R. Vargas, V.: Hydrodynamic Turbulence and Intermittent Random Fields, Communications in Mathematical Physics, 284 (3) (2008), 649-673.

[55] Robert, R., Vargas, V.: Gaussian Multiplicative Chaos revisited, Annals of Probability, 382 (2010), 605-631.

[56] Schramm O. Sheffield S.: A contour line of the continuum Gaussian free field, Probability Theory and related Fields (2012).

[57] Sheffield S.: Gaussian free fields for mathematicians, Probab. Th. Rel. Fields, 139:521-541, 2007.

[58] Sugino F., Tsuchiya O.: Critical behavior in $c=1$ matrix model with branching interactions, Mod. Phys. Lett. A9 (1994) 3149-3162. 